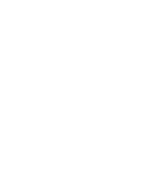
ANNUAL Further
REVIEWS

Click here for quick links to Annual Reviews content online, including:

- Other articles in this volume

- Top cited articles

- Top downloaded articles

- Our comprehensive search

\title{
Mammalian Sperm Motility: Observation and Theory
}

\author{
E.A. Gaffney, ${ }^{1,3}$ H. Gadêlha, ${ }^{1,3,5}$ D.J. Smith,,${ }^{2,3,6}$ \\ J.R. Blake, ${ }^{2,3}$ and J.C. Kirkman-Brown ${ }^{3,4}$ \\ ${ }^{1}$ Centre for Mathematical Biology, Mathematical Institute, University of Oxford, \\ Oxford OX1 3LB, United Kingdom; email: gaffney@maths.ox.ac.uk \\ ${ }^{2}$ School of Mathematics, University of Birmingham, Birmingham B15 2TT, United Kingdom \\ ${ }^{3}$ Centre for Human Reproductive Science, Birmingham Women's NHS Foundation Trust, \\ Birmingham, United Kingdom \\ ${ }^{4}$ Clinical and Experimental Medicine, University of Birmingham, Birmingham B15 2TG, \\ United Kingdom \\ ${ }^{5}$ The CAPES Foundation, Ministry of Education of Brazil, Brasilia, Brazil \\ ${ }^{6}$ School of Engineering and Centre for Scientific Computing, University of Warwick, \\ Coventry CV4 7AL, United Kingdom
}

Annu. Rev. Fluid Mech. 2011. 43:501-28

The Annual Review of Fluid Mechanics is online at fluid.annualreviews.org

This article's doi:

10.1146/annurev-fluid-121108-145442

Copyright (c) 2011 by Annual Reviews. All rights reserved

0066-4189/11/0115-0501\$20.00

\section{Keywords}

flagellum, microswimmer, fluid-filament interactions, slender-body theory

\section{Abstract}

Mammalian spermatozoa motility is a subject of growing importance because of rising human infertility and the possibility of improving animal breeding. We highlight opportunities for fluid and continuum dynamics to provide novel insights concerning the mechanics of these specialized cells, especially during their remarkable journey to the egg. The biological structure of the motile sperm appendage, the flagellum, is described and placed in the context of the mechanics underlying the migration of mammalian sperm through the numerous environments of the female reproductive tract. This process demands certain specific changes to flagellar movement and motility for which further mechanical insight would be valuable, although this requires improved modeling capabilities, particularly to increase our understanding of sperm progression in vivo. We summarize current theoretical studies, highlighting the synergistic combination of imaging and theory in exploring sperm motility, and discuss the challenges for future observational and theoretical studies in understanding the underlying mechanics. 


\section{INTRODUCTION}

Axoneme:

a phylogenetically conserved structure within the eukaryotic flagellum consisting of a ring of nine microtubule doublets and a central pair, frequently referred to as $9+2$

Flagellum: a motile cellular appendage that drives the swimming of sperm and other cells; this article focuses on the eukaryotic flagellum
Sexual reproduction requires the unification of male and female gametes. In mammals, one of the most remarkable events in this process is the journey of the sperm to the egg, occurring through the active swimming behavior of the highly specialized male gamete. Successful sperm, initially part of a population of hundreds of millions, migrate thousands of times their own body length, typically through a complex interior geometry, often filled with highly viscous, non-Newtonian liquids and potentially hostile immune cells. The overwhelming majority do not even reach the fallopian tubes, let alone the site of fertilization.

Although mammalian sperm have many features in common with their evolutionary ancestors in marine invertebrate species such as sea urchin (most notably, the mechanically active axoneme of the flagellum), they migrate through a physical environment that is profoundly different both physically and chemically. Figures $1 a, c$ illustrate the effects of the differences in fluid rheology on the human sperm flagellar beat pattern, with Figure $1 c$ exemplifying the characteristic meandering waveform in the distal (rear) flagellum that human sperm exhibit in the viscid fluids of the female reproductive tract. It is quite remarkable that the progressive velocity of human sperm in such environments, with viscous drag over 100 times that of water, is similar to their swimming speeds within watery, in vitro fertilization media (Smith et al. 2009c). The effect of viscid environments on marine sperm motility is markedly different, with significant reductions in swimming speed (Brokaw 1966, Woolley \& Vernon 2001), yet most of our understanding of sperm motility is based on sea urchin species such as Strongylocentrotus purpuratus and Arbacia punctulata, which are more convenient to study both experimentally and theoretically. This is set against a background in which human fertility is a subject of growing medical and economic importance.

Approximately one in six couples is subfertile, with male factors being present in around half of all cases (Barratt et al. 2009). Although sperm count is a highly publicized measure of fertility, it does not always correlate with adequate motility (Turner 2003). Since Steptoe \& Edwards (1978), powerful interventions involving in vitro fertilization have been available that bypass the need for normal sperm motility; however, these treatments are highly invasive and carry significant risk for the healthy female partner, in addition to being economically prohibitive for many. Conversely, unwanted pregnancy is a major world health and economic problem, but there is no contraceptive currently in clinical use that specifically targets sperm motility. These issues emphasize the importance of scientific research into mammalian sperm motility (Barratt et al. 2009), an area that is currently benefitting from advances in experimental imaging, molecular biology, and computational analysis. It is anticipated that such developments will provide an enhanced foundation for future modeling studies, especially because there has also been a resurgence in explorations of the fundamental biophysics and fluid dynamics of sperm swimming (for reviews and recent research, see Fauci \& Dillon 2006, Riedel-Kruse et al. 2007, Brokaw 2008, Lauga \& Powers 2009, Teran et al. 2010, Mitchison \& Mitchison 2010, Powers 2010).

The theoretical framework for sperm mechanics was initiated with investigations of idealized microswimmers by Taylor $(1951,1952)$ and flagellated cells by Hancock (1953), with Gray \& Hancock (1955) integrating theoretical and experimental studies to explain the basic mechanism of sea urchin sperm motility. This early work has since motivated the development of slenderbody theory for Stokes flow (Lighthill 1976, Johnson 1980) and has influenced later advances such as the boundary integral method (Youngren \& Acrivos 1975) and regularized stokeslets (Cortez 2001). Swimming on cellular scales is counterintuitive and differs in a number of ways from highReynolds number motility (Childress 1981); these include a highly localized flow (Figures $\mathbf{1} \boldsymbol{b}, \boldsymbol{d}$ ), the influence of boundaries on the flow field, and the requirement of kinematic irreversibility for swimming in Newtonian fluids, as demonstrated by Taylor (1960) and epitomized by Purcell's 


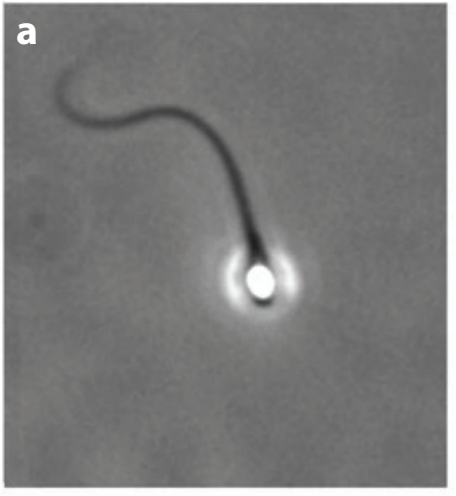

Velocity field magnitude $\left(10^{-4} \mathrm{~m} \mathrm{~s}^{-1}\right)$
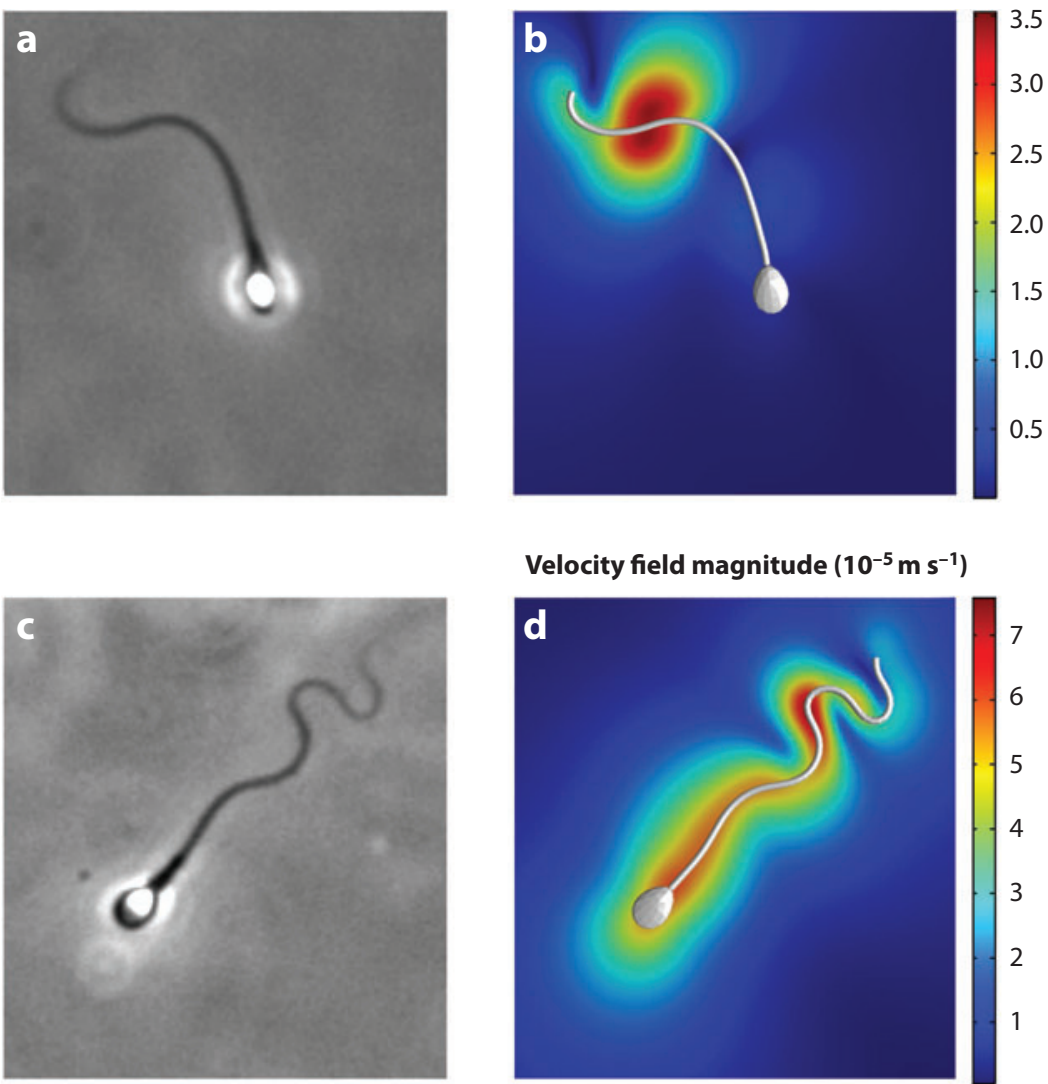

Velocity field magnitude $\left(10^{-5} \mathrm{~m} \mathrm{~s}^{-1}\right)$

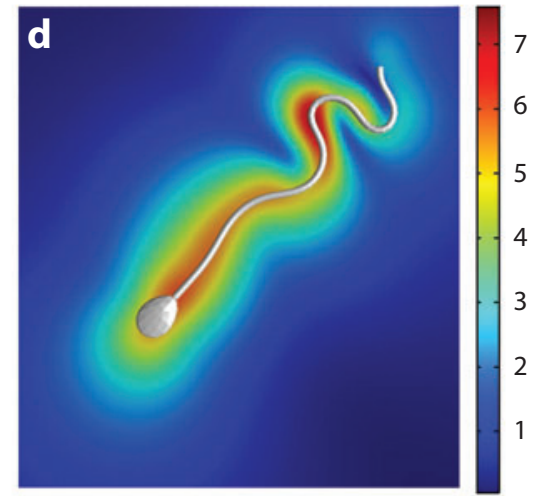

Figure 1

Micrographs, part of a sequence captured at 330 frames per second, and associated fluid mechanical calculations of human sperm migrating in low- and high-viscosity fluids: $(a, b)$ low-viscosity saline medium, with a viscosity of approximately $0.0007 \mathrm{~Pa} \cdot \mathrm{s}$ and $(c, d)$ high-viscosity saline-methylcellulose medium, with viscosity raised to $0.14 \mathrm{~Pa} \cdot \mathrm{s}$. Remarkably, the sperm swims at approximately the same velocity in either medium, despite the large ratio of viscosities. The fluid mechanics calculations were performed using a boundary element/slender-body theory method similar to that implemented by Smith et al. (2009b), which assumes Newtonian fluid properties; the development of full fluid dynamics models taking into account non-Newtonian rheology is discussed in Section 4.2. The computed flow velocity magnitude $|\mathbf{u}|$ is shown at $z=12.5 \mu \mathrm{m}$ above a plane no-slip surface. For both calculations, the simulated cells are located at $z=15 \mu \mathrm{m}$ above the surface. Note the different scalings used for the color axes; the flow-field disturbance is an order of magnitude greater in low-viscosity medium.

(1977) scallop theorem. Consequently, a theoretical basis is required to fully exploit empirical studies of cellular swimming, necessitating a multidisciplinary approach, as originally exemplified by Gray \& Hancock (1955) and by numerous later explorations (Rikmenspoel 1965, 1984; Kinukawa et al. 2005; Riedel-Kruse et al. 2007; Friedrich et al. 2010).

In this review we highlight how fluid dynamics and continuum mechanics may be utilized in conjunction with empirical observations to explore and test our understanding of mammalian sperm motility. In particular, mammalian reproductive biology provides a wealth of unsolved problems for which mechanistic understanding may be beneficial. We must, however, be selective 
Dynein: a molecular motor within the axoneme, attached between adjacent microtubule doublets, that exerts a shearing force to induce axonemal bending and thus neglect some fascinating and important phenomena, such as the roles of cooperativity and competition in sperm dynamics (Immler et al. 2007); the optimization of flagellar swimmers in terms of kinematics and morphometrics (Tam 2008, Spagnolie \& Lauga 2010); models of intracellular signaling during sperm motility (Olson et al 2010); and molecular motor simulations of the flagellum's internal contractile elements, dyneins, and their self-organization (Brokaw 1975, Hines \& Blum 1979, Julicher \& Prost 1997). Section 2 summarizes the basic structure and function of mammalian sperm, with a focus on dynamically important characteristics and their relation to sperm motility and mechanics. We proceed to discuss the mechanically relevant features of the in vivo environment of the female reproductive tract in Section 3, with a review of theoretical models in Newtonian and viscoelastic fluids in Section 4. The final sections summarize recent work bringing together high-speed digital imaging and computational modeling, culminating in a discussion of areas in which the synergy of observation and theory may provide new insights into the fundamental process of sperm migration.

\section{HUMAN AND MAMMALIAN SPERM MORPHOLOGY AND ULTRASTRUCTURE}

\subsection{Eutherian Sperm Dimensions and Beat Frequencies}

There is a remarkable morphological variation of sperm even within the placental (eutherian) mammals: Flagellar lengths range from approximately $35 \mu \mathrm{m}$ for the hippopotamus to $260 \mu \mathrm{m}$ for the Chinese hamster (Cummins \& Woodall 1985). Similarly, rodent sperm typically have a falciform (sickle-shaped) head, whereas the sperm of other species, including humans, have heads in the form of flattened ellipses, with detailed cell morphologies, varying between and even within individuals, highlighting a correlation between genetics and sperm morphology (Austin 1995). Human sperm typically have a head length of $5 \mu \mathrm{m}$ and a flagellum length of approximately $L \sim 55 \mu \mathrm{m}$; although dependent on numerous factors, such as media viscosity $\mu$, the human flagellar beat frequency is typically observed to be approximately $10-20 \mathrm{~Hz}$, which corresponds to an angular frequency of $\omega \sim 60-120 \mathrm{rad} \mathrm{s}^{-1}$ (Yeung et al. 1994, Smith et al. 2009c). For a given

\section{Figure 2}

Illustration of a mammalian sperm cell with the cell membrane removed, revealing the underlying structure including the details of the axoneme. (a) Regions of the sperm flagellum, with representative approximate human sperm length scales indicated (Cummins \& Woodall 1985, Curry \& Watson 1995). The acrosomal cap of the sperm head and connecting piece are also highlighted. (b) A cross section of the mid-piece flagellum (looking from the sperm head to the distal, rear, tip), featuring the presence of nine outer dense fibers immediately exterior to the microtubule doublets, a characteristic feature of internal fertilizers. The doublets and their associated fibers are labeled with the standard convention, clockwise from 1 to 9 , with the first doublet and fiber defined by the radial vector in the direction perpendicular to both the centerline and the separation of the two central microtubules. This structure is also illustrated in panel $e$. The detailed geometry of the outer dense fibers is species specific although fibers 1,5 , and 6 usually have larger cross sections, whereas fibers 3 and 8 are typically the shortest. All fibers taper and end prior to the distal tip of the sperm, are embedded in the connecting piece, and are also bound to their associated doublet at their distal end along the flagellum. In the principal piece of the sperm flagellum (a), there is a fibrous sheath with circumferential ribs, and two longitudinal columns initially attached to fibers 3 and 8 . On moving distally, the ribs of the fibrous sheath become thinner and the columns taper; in addition, the columns appear to be attached to microtubule doublets 3 and 8 distally beyond the termination of the outer dense fibers 3 and 8 , as depicted in panels $c$ and $f$. The end of the fibrous sheath delimits the principal piece from the end piece, where only the axoneme structure persists, as depicted in panels $d$ and $g$. The axoneme structure is further schematically illustrated in panels $b$ and $i$; in the former the microtubules are depicted with their nexin bridges and radial links, whereas the latter illustrates the inner and outer dynein arms, which exert a shearing force between the microtubule doublets. This force bends the microtubules and their associated accessory structures, driving the flagellar waveform. Figure adapted from Fawcett (1975), copyright 1975, and Olson \& Linck (1977), copyright 1977, with permission from Elsevier (2283601155396 and 2370901182457, respectively). 
beat pattern, the flagellum velocity and progressive velocity scale as $\omega L$, with power consumption scaling as $\mu \omega L^{3}$ (Dresdner \& Katz 1981).

\subsection{The Sperm Head}

The human sperm head is packed with haploid DNA and expresses numerous surface receptors and proteins (Figure $2 a$ ). Except in rare pathologies, such as globozoospermia, which imparts sterility (Dam et al. 2007), mammalian sperm do not possess spherical heads. As discussed in Section 4.3.2,
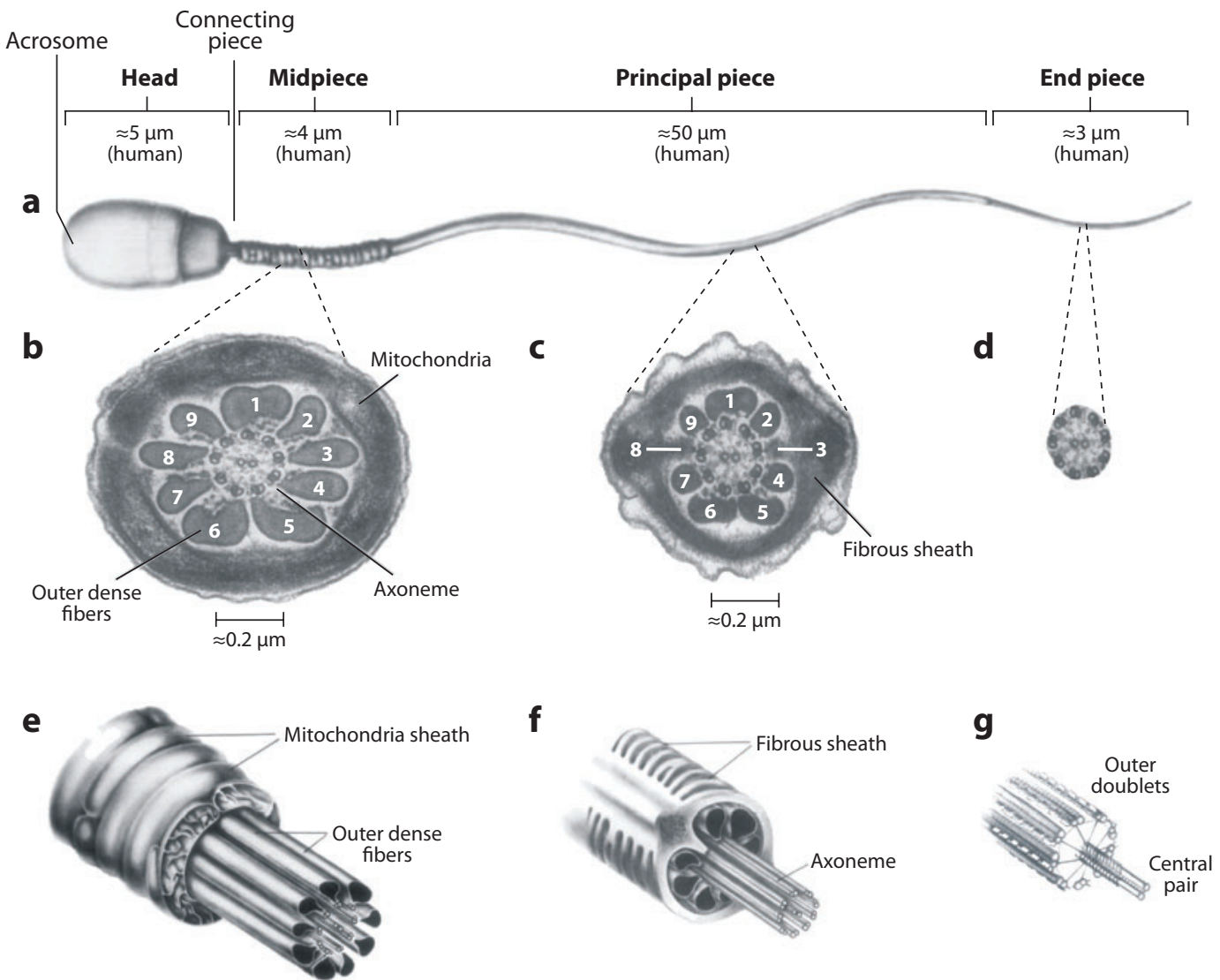

h
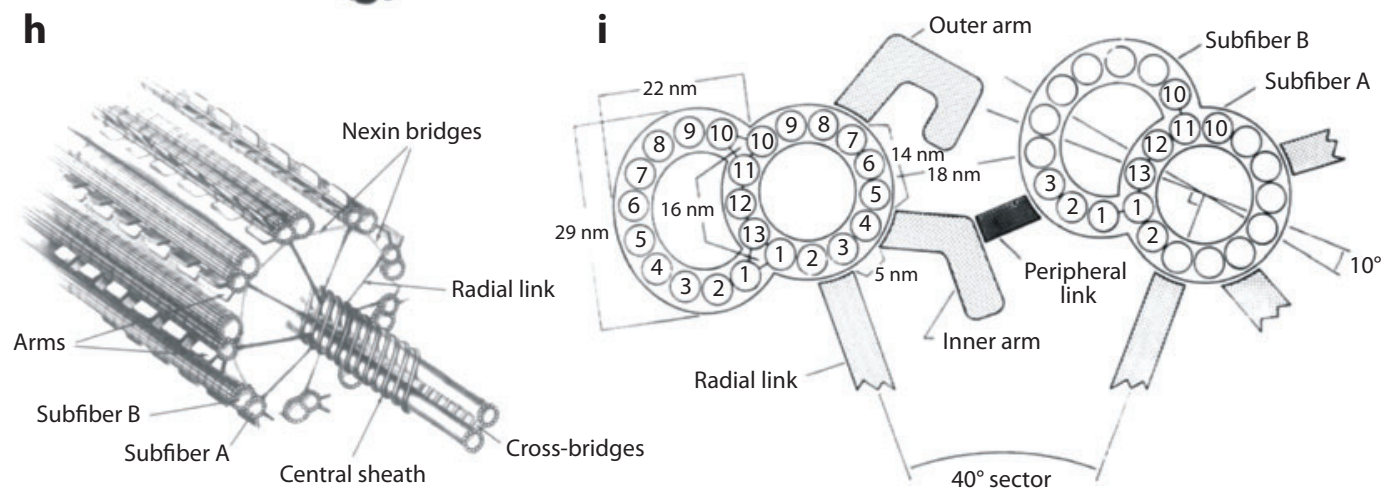
Mid-piece: the region of a sperm flagellum with a mitochondrial sheath, where ATP is generated

\section{Adenosine} triphosphate (ATP): the currency unit of chemical energy transfer in living cells

Microtubule doublet: a pair of proteinaceous filament structures running the length of the axoneme; dyneins drive their bending, which induces flagellar motion

Central pair: a pair of microtubules along the length of the axoneme, symmetrically and slightly offset from the axoneme centerline

Outer dense fibers and fibrous sheath: accessory structures reinforcing the mammalian sperm flagellum; the combined axoneme and accessory structures are referred to as $9+9+2$ sperm have a pronounced tendency to accumulate and swim near surfaces. Comparative studies among species report that head geometrical asymmetry is highly important in surface swimming (Katz \& Phillips 1986, Woolley 2003), as the viscous torques induced by head rotation near boundaries will be contingent on its geometry and orientation. Whether the extensive variation in sperm head shapes among different species of mammalian sperm confers specialized mechanical advantages has not been studied, although the falciform heads of rodent sperm are anticipated to encourage cooperativity in collective swimming, especially in viscous liquids (Immler et al. 2007). Furthermore, theoretical studies indicate that a sufficiently large sperm head stabilizes surface swimming trajectories (Smith \& Blake 2009), whereas Gillies et al. (2009) have shown that head elongation, for a fixed volume, confers a slight swimming advantage in Newtonian liquids, although this results not from a reduction in hydrodynamical drag, but instead from a decrease in cell yawing. The head is densely packed with genetic material, entailing that sperm are not uniformly neutrally buoyant. This suggests a role for geotaxis (Katz \& Pedrotti 1977), although detailed observations have revealed that such effects are usually negligible for human sperm (Winet et al. 1984).

\subsection{The Sperm Flagellum}

The mammalian sperm flagellum (depicted in Figure $2 \boldsymbol{a}$ ) is a motile appendage driven by molecular motors, the dyneins, that induce shear and bending, ultimately leading to cell swimming. Below we explore this structure in detail, emphasizing features of mechanical and dynamical importance.

2.3.1. Passive elements. The sperm head and flagellum meet at the connecting piece (see Figure $2 a$ ). Although the motion of the connecting piece is often too subtle to observe, its elastic compliance has been explicitly documented for chinchilla sperm (Vernon \& Woolley 2004) and has been postulated to be responsible for flagellum beat initiation and alternating directions of bends propagating down the beating flagellum (Vernon \& Woolley 2004, Woolley et al. 2008).

As shown in Figures $2 a, b, e$, the mid-piece of the flagellum is surrounded by a mitochondrial sheath, where adenosine triphosphate (ATP) is generated, contributing in part to the flagellum's energy requirements. Along the centerline of the flagellum interior is a phenotypically conserved and remarkable structure, the axoneme. This consists of a central pair of microtubules symmetrically offset slightly from the flagellum centerline and surrounded by nine pairs of microtubule doublets arranged concentrically around the central pair (Figures $\mathbf{2} \boldsymbol{d}, \boldsymbol{g}, \boldsymbol{b}$ ), forming what is known as the $9+2$ structure. Radial links connect the central microtubule pair to each surrounding microtubule doublet, and nexin bridges connect adjacent doublets.

A crucial difference between marine species that fertilize in low-viscosity seawater and internally fertilizing mammals is the presence of accessory structures attached to the microtubule doublets. In particular, the outer dense fibers and the fibrous sheath are attached to the nine exterior microtubule doublets, forming the $9+9+2$ structure (see Figures $\mathbf{2} \boldsymbol{b}, \boldsymbol{c}, \boldsymbol{e}, \boldsymbol{f}$ ).

In addition, all of this flagellar structure is enveloped within a plasma membrane. A detailed review of the mammalian sperm structure, based on electron microscopy, can be found in Fawcett $(1975,1981)$, while quantitative observations at this resolution are now possible, with rapid sample preparation, using atomic force microscopy (Ierardi et al. 2008).

2.3.2. Active elements. Using an elastic/viscous balance argument, Machin (1958) demonstrated that active force must be generated throughout the flagellum rather than being localized to the head-flagellum junction. This differs from the bacterial flagellum, which is driven entirely by a torque motor at its base. This active force arises from the action of inner and outer arm dynein 
motors, situated on the A subfiber of each doublet, and engaging with the B subfiber of the adjacent doublet (Figure 2i) to exert a sliding force between the doublets.

Following the numbering presented in Figures $\mathbf{2} \boldsymbol{b}, \boldsymbol{c}$, which applies to both the outer dense fibers and their associated microtubule doublets, it has been observed that in the absence of structural constraints, dyneins drive doublet $N$ headward relative to the doublet $N+1$ (Sale \& Satir 1977), with the exception of doublets 5 and 6, which appear to be tightly bound. The associated outer dense fibers, 5 and 6 in the numbering scheme, appear tightly bound in rat (Olson \& Linck 1977), and this is considered to be representative of mammalian sperm (Lindemann et al. 1992). Furthermore, as exemplified by rat studies, observations of the planar beating mammalian sperm flagellum indicate that the microtubule doublets 4-5-6-7 and their outer dense fibers behave as a single functional unit with a permanent linkage between the 5 and 6 elements. Similarly, doublets 9-1-2 and their outer dense fibers respond as a functional unit during mammalian sperm planar beating. The central pair of microtubules together with doublets 3,8 and their accessory structures (including the fibrous sheath, which is exclusively bound to these doublets) form a stiff partition of the flagellum that resists bending. Thus, for example, sliding between microtubules 7 and 8 has the tendency to drive bending in one direction, with sliding between microtubules 2 and 3 driving bending in the other direction, within a beat plane normal to the axis through microtubules 3,8 and the central pair (Lindemann et al. 1992).

Detailed explorations of the mechanisms by which the sliding forces induced by the dynein molecular motors, coupled with the structure of the flagellum, result in the flagellar beat pattern have been the subject of an extensive modeling program (Hines \& Blum 1978, 1979, 1983, 1984, 1985; Murase et al. 1989; Brokaw 1971, 1972, 1984, 1994, 1999, 2002, 2009; Lindemann 1994a,b, 1996, 2002, 2004, 2009; Leisch et al. 2008; Riedel-Kruse et al. 2007). Lindemann's (1996) bull sperm model is particularly noteworthy for mammalian sperm studies, as it focused on documenting the mechanical role of the outer dense fibers on the beating of a pinned flagellum. This collected body of work has been extremely successful in providing insight. For example, detailed comparisons between theory and observation strongly indicate that the inner and outer dynein arms (Figure 2i) differ in their force generation and mechanical roles (Brokaw 1994, 1999; Lindemann 2004). Furthermore, these studies have suggested testable predictions such as the prospect of nanoscale oscillations in the axoneme diameter during flagellar beating, which has since been validated (Lindemann 2004, Lindemann \& Mitchell 2007).

Other questions are immediately apparent. For example, can modeling studies reveal any mechanical advantages or consequences arising from the presence of the fibrous sheath? Does increasing the compliance of the connecting piece of the head-tail junction enhance the asymmetry of the flagellar beat, as suggested by Woolley et al. (2008)? Three-dimensional aspects of the beat pattern and the extent of planarity within flagella models, which are not explicitly constrained to planar beating, are not fully understood and invoke great interest (Brokaw 2003), especially concerning how such features may depend on structural constraints. Furthermore, with the notable exception of Riedel-Kruse et al. (2007), previous modeling studies have considered either a pinned flagellum or a headless flagellum in a quiescent fluid. They also rely on resistive-force theory (see below) to capture the fluid-structure interaction; although this approach is often satisfactory (Johnson \& Brokaw 1979), it can be problematic for nontrivial flagellar interactions that occur in the presence of boundaries and high flagellar curvature. There is a need for investigations that address these interactions and more generally explore how swimming sperm respond through changes in their beat cycle to environmental effects such as alterations in fluid rheology, encountering a shear flow or confronting a boundary. Furthermore, many of these influences are also likely to be important for prospective assisted fertilization and sperm-sorting microdevices (Seo et al. 2007, Shao et al. 2007, Lopez-Garcia et al. 2008). 


\section{HUMAN AND MAMMALIAN SPERM FUNCTION WITHIN THE FEMALE REPRODUCTIVE TRACT}

Oocyte: the egg

It has been observed that "mammalian sperm are introduced into the female reproductive tract in an infertile state and must be educated there before they are able to fertilize oocytes [eggs]" (Florman et al. 2007). Moreover, sperm are unusual cells in that they have limited or no ability to translate proteins (Barratt et al. 2009) and so are reliant on their environment for regulation. Mechanically, the tract environment is rheologically and geometrically complex and is subject to ambient fluid flows induced by peristalsis and cilia. Biochemically, the tract is characterized by a complex milieu of hormones and other species such as nitric oxide, changes in $\mathrm{pH}$ and osmolarity, and the additional challenge of a leukocyte cell invasion induced by sperm. Studies considering even a fraction of these effects are prohibitively difficult in the clinical or research setting, so it would be beneficial to determine when and how it is possible to infer in situ behavior from in vitro assays (see the sidebar for further details). The ultrastructure described above enables vigorous swimming, and hence traversing of the female tract and penetration of the layers surrounding the egg, although only a tiny fraction of sperm ever even approach the female gamete-in humans an initial population of up to hundreds of millions is whittled down to the order of ten or fewer sperm in the vicinity of the egg. Figure 3 depicts the human female reproductive tract, and below we describe some features of migration within this environment, and its mammalian analogs, where mechanical considerations are relevant; such topics are also reviewed by Suarez (2010).

\subsection{Semen Deposition, the Vagina and the Cervix}

The site of semen deposition is species-specific, although in humans semen and sperm are ejaculated at the cervical opening and thus encounter hostile vaginal $\mathrm{pH}$ and immune responses (Suarez \& Pacey 2006), after which rapid sperm accumulation at the cervical mucus boundary is typically observed (Sobrero \& MacLeod 1962). Penetrating the cervical mucus is a substantial barrier to sperm migration; it depends on seminal enzymes, external forces due to visceral contractivity (Katz et al. 1989), and the hydration of the mucus, which varies with the menstrual cycle (Wolf et al. 1978), as well as sperm numbers and motility. Yudin et al. (1989) reported that cervical mucus was compacted at its borders and suggested the consequent greater initial resistance to sperm penetration would explain the initial delays in mucus penetration observed by Cummings et al. (1984). An alternative, untested, hypothesis is that Cummings et al.'s (1984) observations may be explained by the possibility that the sperm flagellum cannot generate as much propulsive force when it is in a less viscous medium at the edge of the mucus border compared to when the sperm has fully penetrated into the mucus.

At the scale of an individual sperm, mucus is not a continuous fluid; rather it is a cross-linked mesh of polymer chains within solution, which the cell must burrow through, compressing and

\section{MOTIVATING THEORETICAL STUDIES OF SPERM MOTILITY}

Laboratory studies of sperm within viscoelastic biological media or background flows are time-consuming, while imaging sperm within complex geometries is fraught with difficulty. In the context of fertility diagnostics or pharmacological screening, such approaches are prohibitively impractical. Thus, advances in the diagnosis and pharmacological modulation of sperm submotility in situ require understanding when it is possible, and how, to accurately infer in vivo behavior from convenient and expedient laboratory analyses of sperm motility. 


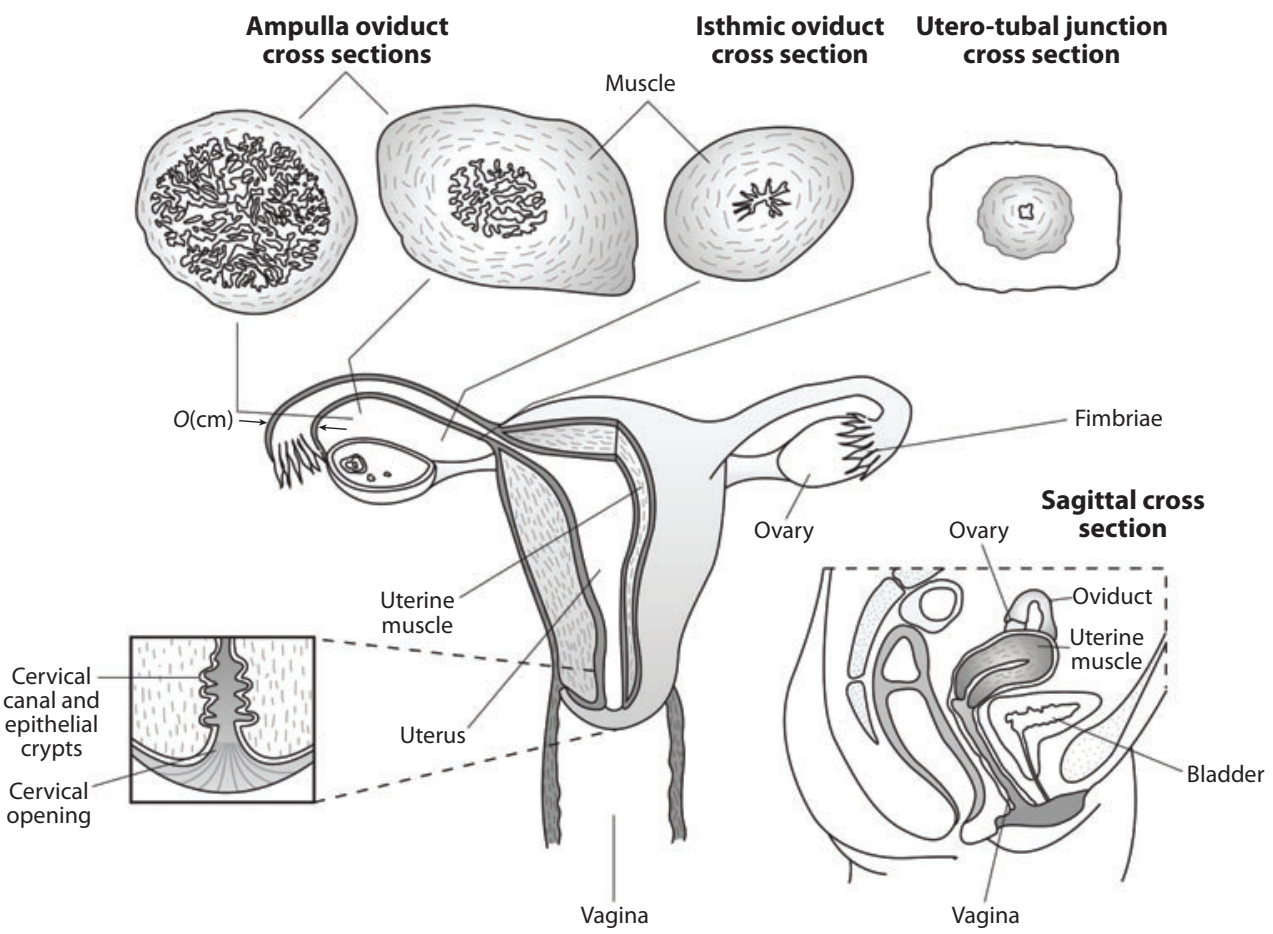

Figure 3

The path of the sperm to the egg within the human female reproductive tract. Up to 300 million sperm are ejaculated at the cervical opening. The interface of the cervical mucus is a substantial barrier to sperm progression with only $0.1 \%$ of sperm colonizing the cervical canal, even under optimal conditions (Croxatto 1995). Migration through the cervix is hindered by a viscous mucus and numerous epithelial crypts, which may possibly act as slow-release reservoirs (Croxatto 1995, Suarez 2002). In contrast, progress through the uterus is understood to be typically rapid and assisted by peristalsis (Kunz et al. 1996). As indicated in the sagittal cross section, the interior of the uterus is a thin film of mucus between two closely opposed surfaces, not an open liquid environment. Passage to the isthmic region of the fallopian tubes (oviducts) proceeds via a narrow lumen, the utero-tubal junction, which is approximately $300 \mu \mathrm{m}$ in diameter and filled with viscoelastic mucus. A continual flux of sperm is observed through the oviducts in fertile human couples around the time of ovulation, although the numbers present at any given time range from less than 10 to a few thousand (Williams et al. 1993); however, there is substantial uncertainty regarding such observations (Croxatto 1995). The oviductal epithelium is lined with motile cilia, interspersed with peg-like secretory cells; as highlighted in the cross sections, there is also a convoluted geometry of epithelial folds within the ampulla regions of the oviducts, where the sperm may encounter the egg. The oviduct diameter in the vicinity of the ovaries is of the order of centimeters, as indicated on the left-hand tube, and the fimbriae move to and fro across the ovaries on the timescale of minutes. Further descriptions of the numerous microenvironments encountered on this journey, and the sperm's response to these, are detailed in the text.

stretching the mucus microstructure, as directly observed by Yudin et al. (1989). Despite such resistance, sperm can propagate remarkably quickly, at speeds of $25-45 \mu \mathrm{m} \mathrm{s}^{-1}$ (Katz et al. 1989), which approaches their swimming motility in lower viscosity semen and in vitro fertilization media, the latter of which has a viscosity approaching that of water. Similar observations can be drawn when considering the viscoelastic mucus analog studies by Smith et al. (2009c), as highlighted in Figure 1. Lindemann's (1996) modeling study of pinned bull sperm strongly indicates that the $9+9+2$ mammalian sperm structure is important in supporting swimming in mucus and 
its analogs, although viscoelastic induced reductions in the flagellar rate of working for a given bending pattern (Fulford et al. 1999) and viscoelastic fluid recoil may also be relevant (Teran et al. 2010).

However, sperm progression through the cervical mucus is not simply a matter of mechanics. The sperm head is coated in a plasma membrane that is biochemically active, and proteins are also expressed in the plasma membrane of the flagellum. Thus, sperm penetration through biological fluids is anticipated to be strongly influenced by electrochemical affinities between sperm surface molecules and the media macromolecules (Katz et al. 1989). These interactions, and variations in surface properties, rather than mechanistic differences, are considered responsible for the observation that slight morphological abnormalities radically alter progression in cervical mucus (Katz et al. 1989). Such influences have been neglected in modeling to date.

Finally, sperm have been observed to be trapped in human cervix epithelial crypts (Croxatto 1995, Hunter 1995), although the mechanics of trapping and release in the cervix is unexplored. While not uniformly accepted, there is the suggestion that this trapping may produce a reservoir, slowly releasing sperm into the reproductive tract over several days (Suarez 2002).

\subsection{The Uterus}

On passing through the cervix, sperm enter the uterus. It is typically understood that sperm should rapidly progress through this region, aided and directed by peristaltic contractions (Kunz et al. 1996), which may also be increased by the release of oxytocin during sexual intercourse (Kunz et al. 2007). However, empirical measurements of peristaltic transport are also reported to be highly variable (for review, see Croxatto 2002, Suarez \& Pacey 2006).

Although generally with the aim of understanding the transport of the embryo postfertilization, mathematical models of peristaltic fluid mechanics in the nonpregnant uterus have been developed (Yaniv et al. 2009); experimental measurements using cine magnetic resonance imaging are also now becoming available (Kido et al. 2008). Consequently, it would be highly informative to investigate whether the current understanding of sperm migration and peristaltic flow is consistent with the transport of sperm to the utero-tubal junctions (see Figure 3). A specific focus could be exploring the biomechanical feasibility of Kido et al.'s (2008) hypothesis that the contraceptive mechanism of intrauterine devices is due to alterations in uterine peristalsis functionally blocking sperm transport.

\subsection{The Utero-Tubal Junctions and the Oviducts}

The sperm must exit the uterus through the utero-tubal junction (Figure 3). For humans and a number of other mammals, this is a narrow lumen filled with viscous mucus (Suarez \& Pacey 2006); once more, sperm penetration of this rheological barrier depends on physicochemical effects as well as fluid mechanics (Nakanishi et al. 2004). The sperm progress from the utero-tubal junction into the isthmic region of the oviducts, which possesses a ciliated epithelium.

For mammals exhibiting estrus such as the cow, binding is readily observed between the sperm head and isthmic epithelium (Suarez \& Pacey 2006). Although the potential functional roles of this binding are the subject of debate (Williams et al. 1993, Croxatto 1995, Hunter 1995, Suarez 2002), there are numerous mechanical questions, such as whether the boundary-accumulating tendency of sperm (see Section 4.3.2) is important in bringing sperm sufficiently close to the epithelium and whether this is influenced by the cilia and background fluid flows. Furthermore, a common question in the biological literature concerns the extent to which observed changes in the beating 
pattern of sperm may enhance epithelial detachment via increased flagellar forces (Suarez \& Pacey 2006) and would benefit from a mechanical analysis.

The changes sperm undergo in preparation for fertilization are known collectively as capacitation (Yanagimachi 1994; for a detailed review, see Suarez 2008). The mechanistic change associated with this is known as hyperactivation; it was originally suggested this may be important in fertilization; however, the timing, triggers, importance, and even the visual characteristics of hyperactivation in vivo are still not fully understood (Barratt \& Kirkman-Brown 2006). At low viscosities, hyperactivation typically manifests as high-amplitude flagellar beating that results in a nonprogressive star-spin or progressive thrashing motility in human sperm (Burkman 1995). Capacitated cells show increased progression through highly viscous and viscoelastic media, which suggests that the hyperactivated state may manifest as enhanced motility in the highly viscous fluid of the isthmus or the cumulus oophorus (Suarez \& Dai 1992). Katz \& Yanagimachi (1980) showed that hyperactivated motility occurs in ex vivo hamster oviduct and is characterized by effective progression through the complicated internal folded geometry (see Figure 3). It has been speculated that hyperactivation may prevent sperm from becoming trapped within the convoluted epithelial folds of the fallopian tubes (Suarez \& Pacey 2006), although other directional cues, such as rheotaxis due to cilia-driven external flows (Katz et al. 1989), are also considered to be potentially important. Determining the mechanical feasibility of these behaviors therefore should be a subject of future studies.

\subsection{Fertilization}

To fertilize, a sperm must penetrate the milieu surrounding the egg. Initially the sperm must traverse the cumulus oophorus, which consists mainly of cells immersed in a hyaluronic gel matrix. In addition to electrochemical affinities, sperm head plasma-membrane enzymes are anticipated to assist the sperm's motility at this stage of its journey (Drobnis et al. 1988), and such enzymes are explicitly found to be expressed on human and murine sperm heads (Lin et al. 1994, Kim et al. 2008). After traversing the cumulus oophorus, the sperm head must then bind to and penetrate a glycoprotein coat, referred to as the zona pellucida. Once bound to this coat, a sperm undergoes the acrosome reaction. Lytic enzymes stored within the acrosome, located at the tip of the sperm head (see Figure $2 \boldsymbol{a}$ ), are released, assisting the sperm in tunneling through to the egg. While it has been suggested that mechanical forces are vital in this process (Bedford 1998), there is still no consensus on the relative importance of mechanics compared to enzyme lysis (Kim et al. 2008). Thus detailed investigations are merited, although preliminary calculations have been pursued (Baltz et al. 1988, Green 1988).

In summary, at almost every stage in the sperm's ascension of the female reproductive tract and in the final approach to the egg, there are numerous biologically important questions that will benefit from additional biomechanical analysis.

\section{SURVEY OF THEORETICAL STUDIES}

\subsection{Newtonian Fluid Mechanics}

We briefly summarize the classical application of Newtonian fluid mechanics to flagellated cell swimming, via slender-body theory and resistive-force theory, noting that this field has been reviewed extensively elsewhere (Lighthill 1976, Brennen \& Winet 1977, Sleigh 1991, Lauga \& Powers 2009).
Capacitation: the physiological state of a sperm required for fertilization, which is accompanied by the motility patterns associated with hyperactivation, characterized in saline by high-amplitude asymmetric beating

Cumulus oophorus: the outer vestment of the mammalian egg consisting of hundreds of cells radiating out from the egg embedded within a non-Newtonian hyaluronic acid gel

Rheotaxis: directed motility in response to the influence of fluid flow

Shear: in the context of the flagellum, the relative movement of adjacent microtubule doublets

Zona pellucida: a tough glycoprotein coat between the human egg and the cumulus oophorus, which a sperm must penetrate for successful fertilization Acrosome: the cap of the sperm head containing enzymes allowing penetration of the zona pellucida via the acrosome reaction

Resistive-force theory: an approximation for the local drag of a slender filament element in Stokes flow (or a viscoelastic generalization thereof) 
Slender-body theory: an improved approximation for the local drag on a slender filament element in Stokes flow (or a viscoelastic generalization thereof)
The length scales and timescales of human sperm swimming are such that the Reynolds number is approximately $10^{-3}$, within the regime of Stokes flows, and thus inertia is negligible. Consequently, the equations of motion for a Newtonian fluid surrounding a sperm are

$$
-\nabla p+\mu \nabla^{2} \mathbf{u}=\mathbf{0}, \quad \nabla \cdot \mathbf{u}=0, \quad \mathbf{x} \in \Omega,
$$

where $p$ is the modified pressure, including the gravitational potential. The parameter $\mu$ is dynamic viscosity, and $\mathbf{u}$ is the velocity vector field in the domain $\Omega$ external to the cell and its flagellum. This is supplemented by the boundary condition $\mathbf{u}=\mathbf{U}$ for $\mathbf{x}$ on the sperm boundary, where $\mathbf{U}$ denotes the speed of the sperm boundary and thus represents no slip. Standard adjustments to the boundary conditions apply when the sperm is in the presence of nontrivial geometries as opposed to free space.

4.1.1. Resistive-force theory. The essential propulsive mechanism for sperm was first given by Gray \& Hancock (1955). Using the resistive-force theory implicit in Hancock (1953), which entails that the drag coefficients associated with normal $\left(C_{N}\right)$ and tangential $\left(C_{T}\right)$ motion satisfy $C_{N} / C_{T} \approx 2$, Gray \& Hancock fundamentally explained how a propagating flagellar wave produces a propulsive thrust that is balanced by the drag on the cell head. In detail, resistive-force theory can be interpreted as a logarithmically accurate local approximation, which treats the ratio of the flagellum radius to its bending radius of curvature as a small parameter (Johnson 1980). In particular, a slender flagellum element of length $\delta s$ with speed $U_{T}$ in its tangential direction and speed $U_{N}$ in its normal direction experiences a local viscous drag approximately given by $-C_{N} U_{N} \delta s$ and $-C_{T} U_{T} \delta s$ in the respective directions, where $C_{N}$ and $C_{T}$ are constant resistance coefficients; they are not equal. This anisotropy emerges from the slenderness of the flagellum and entails that even a symmetric flagellum beat will not have forces and torques cancelling by symmetry. The net force and torque exerted by the sperm flagellum are instead balanced by the viscous drag of the sperm head. The latter varies with the translational and angular velocity of the head; thus the force and torque balance yields the sperm's velocity and angular velocity in terms of the flagellar beat waveform. Such calculations are analytically tractable and compare favorably with observations for planar flagellar waveforms (Gray \& Hancock 1955, Dresdner \& Katz 1981, Friedrich et al. 2010).

4.1.2. Slender-body theory. In free space, or simple geometries such as half-space, a more accurate approximation utilizing slender-body theory for the flagellum can be readily developed. Considering a headless flagellum, an ansatz for the solution of Equation 1 is to place a weighted line distribution of Stokes flow singularities (Pozrikidis 2002) along the centerline of the flagellum. Thus one considers

$$
\begin{aligned}
u_{i}^{\text {Ansatz }}(\mathbf{x}) & =\int_{\text {Flagellum }} d s G_{i j}(\mathbf{x}, \mathbf{X}(s)) f_{j}^{\text {hyd }}(s)+\int_{\text {Flagellum }} d s D_{i j}(\mathbf{x}, \mathbf{X}(s)) g_{j}^{\text {hyd }}(s), \\
G_{i j} & =\frac{1}{8 \pi \mu}\left(\frac{\delta_{i j}}{R}+\frac{R_{i} R_{j}}{R^{3}}\right), \quad D_{i j}=\frac{1}{4 \pi}\left(-\frac{\delta_{i j}}{R^{3}}+3 \frac{R_{i} R_{j}}{R^{5}}\right), \quad \mathbf{R}=\mathbf{x}-\mathbf{X}(s), \quad R=|\mathbf{R}| .
\end{aligned}
$$

For Newtonian fluids, the equations have no explicit time dependence, and hence we omit the parameter $t$ for brevity in this section. The point $\mathbf{X}(s)$ is the flagellum centerline at arc length $s$ (see Figure 4). One can confirm that the above integral satisfies Equation 1, with the pressure field defined analogously. Although $\mathbf{u}^{\text {Ansatz }}$ decays at spatial infinity, it will not satisfy the required boundary conditions in general. Imposing these conditions for $\mathbf{x}$ on the flagellum surface yields constraints for the unknown singularity weightings, $\mathbf{f}^{\text {byd }}(s)$ and $\mathbf{g}^{\text {byd }}(s)$, in the form of integral equations. 


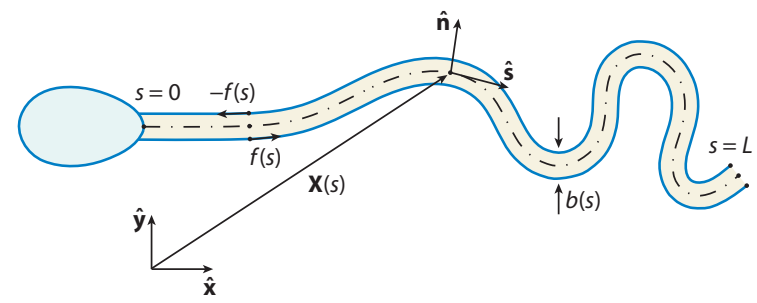

Figure 4

The sperm flagellum for a planar waveform at a fixed time, $t$. The vector $\mathbf{X}(s)$ represents the location of the flagellum centerline at arc length $s$. The tangent vector is denoted $\hat{\mathbf{s}}=\mathbf{X}_{s}$, the normal is given by $\hat{\mathbf{n}}=\mathbf{X}_{s s} /\left|\mathbf{X}_{s s}\right|$, and the binormal $\hat{\mathbf{b}}=\hat{\mathbf{t}} \wedge \hat{\mathbf{n}}$ points out of the figure. The flagellum length is $L$, and the axoneme diameter is $b(s)$, which can vary (Lindemann \& Mitchell 2007). Furthermore, $f(s)$ denotes the total, active and passive, sliding force driving the two structural halves of the flagellum within the beat plane, whose normal direction is given by the axis through the central pair, and the 3,8 microtubule doublets (Lindemann et al. 1992). Points on the flagellum within the beat plane are given by $\mathbf{r}_{ \pm}(s)=\mathbf{X}(s) \pm$ $b(s) \mathbf{n}(s) / 2$, and the amount of shear at arc length $s$ is then given by $\Delta(s)=\Delta(0)+\int_{0}^{s}\left\{\left|\mathbf{r}_{-}^{\prime}(s)\right|-\left|\mathbf{r}_{+}^{\prime}(s)\right|\right\} d s$. Here the primes denote differentiation with respect to arc length, and $\Delta(0)$ represents shearing at the connecting piece, commonly referred to as basal shearing. $\Delta(s)-\Delta(0)$, denoted as the differential shear, is the amount of shear exerted on the flagellum at arc length $s$.

Hancock's (1953) planar slender-body theory and associated resistive-force theory utilize the dipole weighting $\mathbf{g}^{\text {hyd }}(s)=-a^{2}\left[\mathbf{f}^{\text {hyd }}(s) \cdot \mathbf{n}\right] \mathbf{n} /(4 \mu)$, where $\mathbf{n}$ is the unit normal to the flagellum in the beat plane, and $a$ is the flagellum radius. An alternative representation is based on modeling the flagellum as a slender ellipsoid with cross-sectional radius $\bar{a}\left(s_{1}^{2}-s^{2}\right)^{1 / 2}$, with $s_{1}$ and $\bar{a}$ constant; then one has $\mathbf{g}^{\text {hyd }}=-\bar{a}^{2}\left(s_{1}^{2}-s^{2}\right) \mathbf{f}^{\text {hyd }} /[4 \mu]$, as derived by Johnson (1980) and used in sperm modeling by Smith et al. (2009b). This has the advantage of greatly improved accuracy near the flagellar tip. A priori, there is no guarantee that a solution of these integral equations exists. Johnson's (1980) detailed calculations nonetheless prove that, given the latter relationship between $\mathbf{f}^{\text {byd }}(s)$ and $\mathbf{g}^{\text {byd }}(s)$, these weightings can be chosen so as to satisfy the boundary conditions to algebraic accuracy in the ratio of the flagellum radius to its bending radius of curvature, even at the end points of the flagellum. Furthermore, once $\mathbf{f}^{\text {hyd }}(s)$ and $\mathbf{g}^{\text {byd }}(s)$ are approximated, one can also generate the flow field to the same level of accuracy. This methodology generates efficient numerical simulations, and the use of half-space Stokes flow singularities (Blake 1971, Pozrikidis 2002) readily allows the exploration of surface effects on flagellar hydrodynamic drag. This framework has been amalgamated with boundary element theory to additionally incorporate the influence of realistic head geometries (Smith et al. 2007, 2009b), and its use is illustrated in generating the flow-field magnitudes of Figures $\mathbf{1} \boldsymbol{b}, \boldsymbol{d}$. For the diffusion coefficients associated with sperm chemoattractants such as progesterone and bourgeonal, convection is important in these flows and dominant for sperm undergoing hyperactivation, as first demonstrated by Dresdner \& Katz (1981). This emphasizes that the flagellar beat cannot be neglected in the transport of signaling molecules close to the cell or the design of sperm chemotactic assays.

4.1.3. Direct-solution techniques. Slender-body theory is invalid, however, for the close interaction of flagella with boundaries, very high flagellar curvature, or the hydrodynamic interaction of two sperm. Under such scenarios, one must currently resort to implementing direct numerical solutions of the fundamental equations given in Equation 1. Immersed boundary and boundary element methods are finding popularity in the simpler setting of the prokaryotic flagellum 
(Cortez et al. 2004, Hsu \& Dillon 2009, Shum et al. 2010), whereas the finite-volume method has also been considered for eukaryotic cilia (Mitran 2007).

\subsection{Non-Newtonian Fluid Mechanics}

Newtonian resistive-force theory had often been utilized to provide an order-of-magnitude approximation to assess sperm mechanics within viscoelastic and biological media (Baltz et al. 1988, Drobnis et al. 1988), motivated by the fact that a suitable viscoelastic theory had not been developed at the time. Observations have since indicated that even the relations between kinematic parameters and swimming speed for sperm in weakly viscoelastic methylcellulose solutions are not explained by Newtonian models (Smith et al. 2009c).

Improving our understanding of sperm motility in the female reproductive tract therefore motivates the development of new modeling paradigms to cope with moving boundary problems in viscoelastic and complex rheologies. Fulford et al. (1999) generalized resistive-force theory to linear viscoelasticity, revealing that the hydrodynamic drag per unit length of flagellum in a Maxwell fluid in the tangential, $f_{T}^{\text {Maxwell }}$, and normal, $f_{N}^{\text {Maxwell }}$, directions satisfies

$\lambda \frac{\partial f_{T}^{\text {Maxwell }}}{\partial t}(s, t)+f_{T}^{\text {Maxwell }}(s, t)=-C_{T} U_{T}(s, t), \quad \lambda \frac{\partial f_{N}^{\text {Maxwell }}}{\partial t}(s, t)+f_{N}^{\text {Maxwell }}(s, t)=-C_{N} U_{N}(s, t)$.

Here $s$ denotes the arc length along the flagellum; $t$ is time; $\lambda$ is the elastic recoil time; $U_{T}$ and $U_{N}$ are, respectively, the tangential and normal components of the flagellar velocity; and $C_{T}$ and $C_{N}$ respectively denote the Newtonian tangential and normal resistance coefficients. Fulford et al. (1999) proceeded to demonstrate that the rate of working is reduced as elasticity is increased for small-amplitude motions in a linear viscoelastic fluid.

More recently, there has been a resurgence of studies exploring the effects of fluid elasticity in microbiofluidics, considering flagellar and cilia beating in linear viscoelastic media (Fu et al. 2008, Smith et al. 2009a), as well as idealized microswimmers (Normand \& Lauga 2008), even in nonlinear viscoelastic fluids (Teran et al. 2010) such as the Oldroyd-B medium (Oldroyd 1950). These studies demonstrate that a substantial revision of our understanding is required in the presence of viscoelasticity. For example, Purcell's scallop theorem is invalidated (Normand \& Lauga 2008), and hydrodynamic drag forces are significantly reduced (Fu et al. 2008). However, full three-dimensional finite-amplitude, finite-length simulations within media with nonlinear viscoelasticity, anisotropy, or other complex rheologies remain to be considered. Finally, we note that modeling motility within physiological mucus has the additional complications of microrheological and electrochemical interactions between the sperm and the mucin fibers (Katz et al. 1989, Lai et al. 2009).

\subsection{Fluid-Structure Interactions}

In isolation, the above techniques (from resistive-force theory to direct numerical simulations) only allow the extraction of hydrodynamic drag forces from observations of sperm. However, the flagellar beat emerges from a balance of hydrodynamic drag, the passive structural forces of the flagellum, and the active forces of the dynein molecular motors. This is illustrated in Figure 1, where viscosity changes substantially affect the flagellar waveform; hence one also needs to explore the interaction of the flagellum with the surrounding fluid.

Brokaw (1972, 1999, 2002, 2003), Lindemann (1994a,b, 2002, 2004), and Leisch et al. (2008) have pursued programs modeling the internal mechanics of the sperm axoneme in detail, primarily 
to understand the mechanism of flagellar oscillation and the development of the waveform. Alternative representations of the flagellum have also been highly instructive in exploring fluid-structure dynamics, especially during sperm swimming. The immersed boundary method, exemplified by Cortez et al. (2004) and reviewed by Fauci \& Dillon (2006), can be used to explore flagellum-fluid interactions, although geometrically accurate representations of flagella have only been considered for prokaryotes (Hsu \& Dillon 2009). Hines \& Blum $(1978,1979)$ initiated a modeling program representing the flagellum as a continuous Euler-Bernoulli filament, with an additional passive response to shearing. This framework was first generalized for swimming filaments with cell bodies by Camalet \& Julicher (2000) and is consistent with recent observations characterizing the shearing stiffness of sea urchin flagella with disabled dyneins (Pelle et al. 2009).

In addition, shearing filament models of flagella may also be considered in terms of Cosserat rod theory (Antman 2005), whereby passive shearing responses are automatically incorporated via the material constitutive relation. Explorations of this conceptually elegant framework are merited to assess whether alternative representations of flagellar elasticity refine the agreement between theory and empirical studies of shearing.

4.3.1. Hydrodynamic drag, filament mechanics, and sliding forces. Models coupling resistive-force-theory hydrodynamic drag, filament mechanics, and sliding forces for a swimming sperm typically treat the flagellum as an Euler-Bernoulli filament, supplemented with the above-mentioned additional passive shearing response, and explore the emergent flagellar beat pattern and its influence on motility (Brokaw 1971, Hines \& Blum 1978, Camalet \& Julicher 2000, Riedel-Kruse et al. 2007, Fu et al. 2008, Hilfinger et al. 2009, Gadêlha et al. 2010). In contrast, one can also use this framework to extract the hydrodynamic forces and the passive filament mechanics from observations, as we now describe.

Here we consider planar beating in the absence of torsion as illustrated in Figure 4; the binormal component of the bending moment per unit flagellum length about $\mathbf{X}(s)$, arising from passive elastic resistance, is given by

$$
m_{\text {elas }}(s)=-\frac{\partial}{\partial s}(E(s) \kappa(s)),
$$

where $E(s)$ is the flagellar bending stiffness, and $\kappa(s)$ is flagellar curvature.

Similarly, the analogous contribution to the bending moment per unit length from both active and passive forces in the sliding filament model is simply given by (Brokaw 1971, Hines \& Blum 1978, Camalet \& Julicher 2000, Riedel-Kruse et al. 2007, Fu et al. 2008, Hilfinger et al. 2009, Gadêlha et al. 2010)

$$
m_{\text {slid }}(s)=b(s) f(s),
$$

where $f(s)$ is the net sliding force, as detailed in Figure 4, and $b(s)$ is the axoneme diameter, which can in general vary (Lindemann \& Mitchell 2007).

Finally, the contribution of hydrodynamic drag to this component of the bending moment per unit length about $\mathbf{X}(s)$ is given by

$$
m_{\text {byd }}(s)=-\hat{\mathbf{n}}(s) \cdot \int_{s}^{L} d s^{\prime} \mathbf{f}^{\text {byd }}\left(s^{\prime}\right),
$$

where $\mathbf{f}^{\text {byd }}(s)$ is the hydrodynamic force per unit length exerted by the fluid on the flagellum at an arc length $s$ along the flagellum. If the beat plane is an equipotential of gravity, or with neutral buoyancy, a moment balance immediately yields

$$
f(s)=\frac{1}{b(s)}\left[\frac{\partial}{\partial s}(E(s) \kappa(s))+\hat{\mathbf{n}}(s) \cdot \int_{s}^{L} d s^{\prime} \mathbf{f}^{b y d}\left(s^{\prime}\right)\right] .
$$


Thus, given basic assumptions and knowledge of the mechanical stiffness of the flagellum, one can estimate the total shear force, active and passive, from observations of the flagellum, which we explore further below. In contrast, if the shear forces are prescribed, then balancing the moments in Equations 3-5 and invoking resistive-force theory to express $\mathbf{f}^{\text {hyd }}$ in terms of the local flagellar velocity generate an equation for the motion of the flagellum. Coupling this with a global force and torque balance for the cell via the boundary conditions for the equations of motion will yield the flagellar waveform and the cell swimming trajectory (see, e.g., Camalet \& Julicher 2000).

4.3.2. Interactions of sperm with boundaries, other sperm, and background flows. It is well-known that sperm are influenced by boundaries and background flows (Rothschild 1963, Winet et al. 1984, Cosson et al. 2003, Woolley 2003), and such mechanisms are likely to be important at various stages in the progression of sperm up the female reproductive tract. Despite the importance of such phenomena being recognized within the fluid dynamical literature (Pedley \& Kessler 1992), relatively little modeling has considered the underlying mechanical principles. Boundary accumulation was numerically investigated for swimming sheets by Fauci \& McDonald (1995) and for realistic sperm geometries by Smith et al. (2009b). Hydrodynamical explanations for the boundary accumulation of bacteria have been reported (Berke et al. 2006), although a detailed consideration of cell geometry is required to additionally differentiate between stable surface swimming, descent into the boundary, and surface escape (Shum et al. 2010). However, such studies do not immediately translate to mammalian sperm due to the manifest differences of the prokaryotic flagellum. Fundamental explanations for sperm swimming next to no-slip boundaries have been explored by Smith \& Blake (2009), but a complete understanding remains elusive. For example, the exquisite sensitivity to variations in the size of the sperm head and the wave number of the flagellum are unexplained, whereas the influence of the boundary on the flagellum beat remains to be documented.

In addition, Woolley et al. (2009) have recorded details of bull sperm flagella synchronizing as they swim in close vicinity, although phase locking was reported only to occur once the sperm heads had come into contact. Flagellar synchronization has been studied in prokaryotic flagellated swimming (Hsu \& Dillon 2009), in eukaryotic cilia metachronal coordination (Gueron et al. 1997, Mitran 2007), and for infinite swimming sheets (Elfring et al. 2010) but has not been extensively considered for swimming sperm. Finally, the influence of background flows on sperm motility has essentially been ignored in the modeling literature to date.

4.3.3. Fluid-structure interactions with viscoelastic media. Theoretical explorations of the interplay among combinations of the above fluid-structure phenomena and, in addition, the influence of viscoelasticity are also important, although studies are in the early stages. The main focus has been how viscoelasticity modulates the formation of a flagellar beat. This has been investigated using linear viscoelasticity (Fu et al. 2008) and via immersed boundary techniques in the simplified setting of swimming sheets (Dillon et al. 2003a,b, Teran et al. 2010). The latter study considered finite swimming sheets with finite-amplitude motions for an Oldroyd-B fluid and demonstrated that elastic effects reduced backward movement during beat recovery and gave optimal progression at $\mathrm{De}=1$, which has close parallels with the characteristic Deborah number of sperm in mucus (see also Smith et al. 2009c). A subtlety in these models is the prescription of the driving mechanism for flagellar bending; explorations of the interaction of viscoelasticity with the regulatory mechanism of real flagella have not been initiated.

4.3.4. Final remarks. Studies of fluid-flagellum interactions for eukaryotes often rely on simplifying assumptions such as a two-dimensional representation or resistive-force theory. The latter 
approximation is used despite the fact that the feedback from fluid drag is also postulated to influence bend propagation (Woolley 2007) and that sperm swim in highly viscous media. Consequently, the use of coupled slender-body filament-fluid models, such as the classical LGL theorem (Liron 2001), which is used extensively in ciliary dynamics, is likely to be important in facilitating further theoretical research of sperm motility. Similar comments apply for direct simulation techniques, as illustrated by the studies of Mitran (2007), which again are for cilia. Such approaches will improve the accuracy of recent studies of complex fluid-structure interactions due to improved representations of hydrodynamic drag. One potential application area is beat pattern formation within the high-curvature flagella characteristic of migrating cells in high-viscosity liquids, and hyperactivated cells in low-viscosity liquids. A second application would be exploring the modulation of a sperm's flagellum beating by its environs (e.g., boundaries, other sperm, or background flows).

\section{COMBINING EXPERIMENTAL IMAGING AND THEORY}

\subsection{Background}

The above discussion highlights numerous areas where theoretical modeling can be used to interpret phenomena observed by biologists. In this section we focus on the synergy between microscopy and theory, which has long been recognized (Gray \& Hancock 1955; Rikmenspoel 1965, 1984), and illustrate how digital capture and analysis techniques, together with mechanics, can inform our understanding of sperm motility.

\subsection{Flagellum Internal Mechanics and Dynein Regulation}

Riedel-Kruse et al. (2007) considered a linearized model of the flagellum beat based on resistiveforce theory and sliding elastic filaments driven by active forces, which are regulated by the extent of the sliding via a self-organization model. This study quantitatively compares modeling predictions with experimental observations of beating bull sperm and concludes that basal sliding of the filaments is required, as observed by Vernon \& Woolley (2004). In contrast to Lindemann's (1996) predictions, based on the geometric clutch hypothesis, Riedel-Kruse et al. (2007) reported that the outer dense fibers and fibrous sheath do not play an important role in shaping the beat pattern. However, this is based on the assumption that the influence of these structures' bending stiffness variation can be treated as a perturbation; in contrast, later measurements reveal that the passive stiffness of the bull sperm flagellum varies substantially along its length (Leisch et al. 2008).

This contrast between different theoretical perspectives illustrates that further studies coupling observations and theory are merited, especially given that the regulation of the flagellar beat pattern is far from completely understood.

\subsection{From Microscopy to Mechanics}

The early study of Yundt et al. (1975) combined experimental imaging using high-speed cine micrography with the fluid dynamic analysis of Gray \& Hancock (1955). Using early digital computer technology, the authors found that data-smoothing procedures were necessary to eliminate experimental noise. The predictions of swimming trajectories resulting from the observed flagellar motions only yielded qualitative agreement with the actual trajectories, a demanding test as errors in velocity and, more seriously, angular velocity are cumulative in time. 
Bending moment density: the moment per unit length associated with flagellar bending; it can be divided into a hydrodynamic moment, an elastic moment (from the flagellar bending stiffness), an active moment (generated by dyneins exerting forces between adjacent microtubule doublets), and a passive moment resisting shear
Since the initial work of Baba \& Mogami (1985), digital imaging and flagellar capture have been used to analyze the motion of the flagellum, allowing much higher-throughput analysis of the resulting time-series data. Whereas some studies have focused on kinematics only (e.g., Ohmuro \& Ishijima 2006, Smith et al. 2009c), a small number have combined the kinematic data with an analysis of the fluid mechanics (e.g., Kinukawa et al. 2005, Riedel-Kruse et al. 2007). In particular, recent explorations by Gillies et al. (2009) and Friedrich et al. (2010) demonstrate an improved quantitative agreement between theory and experiment in predicting swimming behaviors from observed flagellar waveforms, thus empirically validating the Newtonian hydrodynamics of sperm swimming to higher accuracies than previous studies.

Here we consider the images emerging from the flagellar-capture experiments of Smith et al. (2009c) in detail, focusing on the planar progressive swimming of normozoospermic human cells in highly viscous, non-Newtonian, $1 \%$ methylcellulose solution [Sigma-Aldrich M0512, graded to give $2 \%$ aqueous solutions with nominal viscosity $4 \mathrm{~Pa} \cdot \mathrm{s}$ at $20^{\circ}$; effective viscosity of $1 \%$ solution $0.14 \mathrm{~Pa}$ at $37^{\circ}$ and frequency $5 \mathrm{~Hz}$ (Smith et al. 2009c)]. Statistical measurements of the kinematics for multiple sperm were considered by Smith et al. (2009c); in the following we focus on the trends that emerge from spatially and temporally resolved data in combination with continuum mechanics.

Figure $5 \boldsymbol{e}$ shows estimates for the total bending moment density due to active and additional passive shearing forces of a swimming sperm, $m_{\text {slid }}(s)=b(s) f(s)$. The axonemal diameter, $b(s)$, is approximately $170 \mathrm{~nm}$ although $O(25 \%)$ fluctuations are observed during flagellar bending (Lindemann \& Mitchell 2007); thus the internal active and passive sliding force density $f(s)$ may also be estimated, subject to the approximation that $b(s) \sim 170 \mathrm{~nm}$. An additional approximation in estimating bending moment densities arises from the out-of-plane motion characterized by the variation of the projected flagellum length by about $\delta L / L \sim 0.1$. By neglecting the binormal velocity, the associated relative error is of the order $(\delta L / L)^{1 / 2} \sim 0.3$, which induces relative errors of up to this size in estimates of the hydrodynamic bending moment density.

A further approximation is the estimate of flagellar bending stiffness as direct measurements are not available for human sperm, although spatially resolved data have been reported for both rat and bull sperm (Schmitz-Leisch \& Lindemann 2004, Leisch et al. 2008). Measurements of the shear stresses that eradicate motile function in comparative studies indicate that simple scaling relations, assuming the same material properties of the outer dense fibers in the mid-piece, give excellent estimates of flagellar response, except for bull and guinea pig, which are anomalous (Baltz et al. 1990). One may therefore estimate human flagellar stiffness by taking data from the rat, assuming fixed material properties and scaling. The human mid-piece outer dense fiber crosssectional area is one-eighth that of the rat (Baltz et al. 1990), yielding the prediction that the human flagellum is one-sixty-fourth as stiff in this region; the absence of quantitative comparative studies of the accessory structures distal to the mid-piece prevents the extension of technique to the whole flagellum. Thus the bending stiffness is also smoothly fitted through the measured sea urchin-flagellum passive bending stiffness of $10^{-21} \mathrm{Nm}^{2}$ (Okuno et al. 1981) in the distal flagellum, where accessory structures are absent as with sea urchin. This fitting is performed with an exponential as this form of decay is the qualitative trend in flagellum stiffness measurements (Schmitz-Leisch \& Lindemann 2004, Leisch et al. 2008). The resulting bending stiffness is plotted in Figure $5 \boldsymbol{b}$, although the qualitative details of the plots are not altered with moderate variations of the bending stiffness, such as doubling $E(s)$ in the distal flagellum. These approximations imply that it is rational to use viscoelastic resistive-force theory rather than more accurate methods to determine the hydrodynamic drag.

The total sliding bending moments in Figure $5 \boldsymbol{e}$ are nontrivial in the basal region of the flagellum, which is necessary for compliance of the connecting piece to be dynamically important, 
a

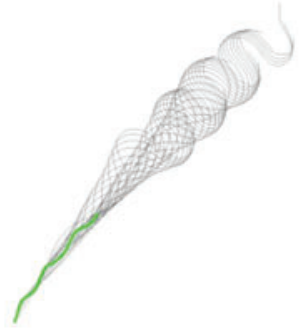

C

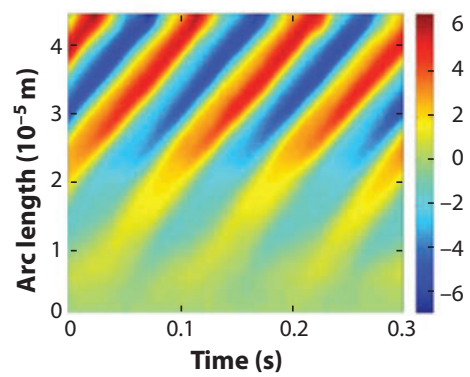

f

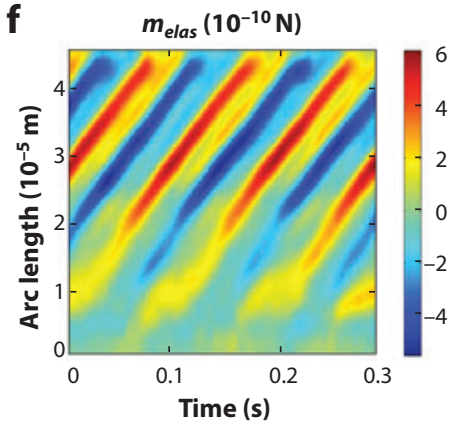

b

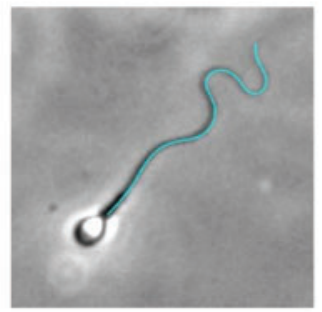

d

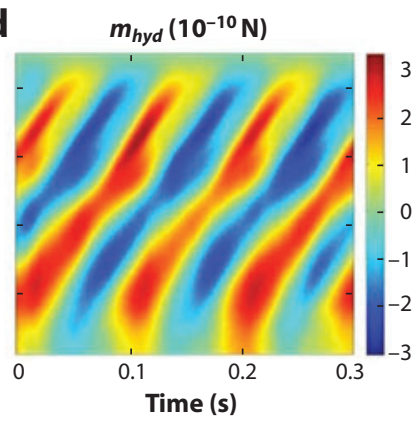

e
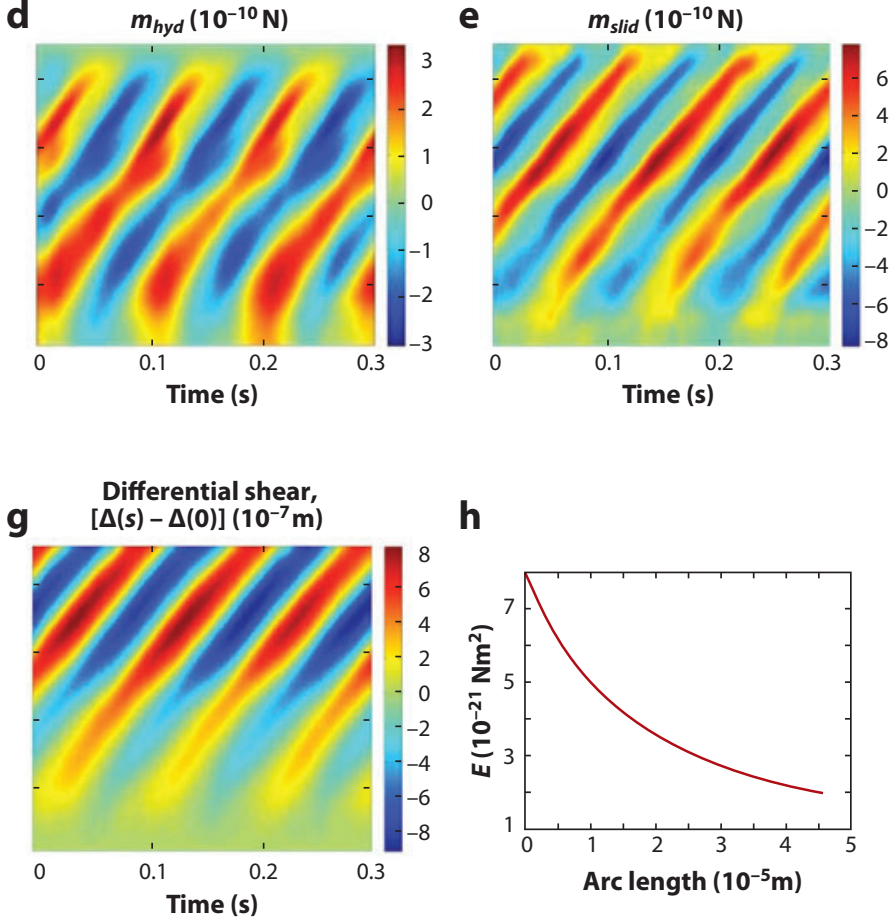

h

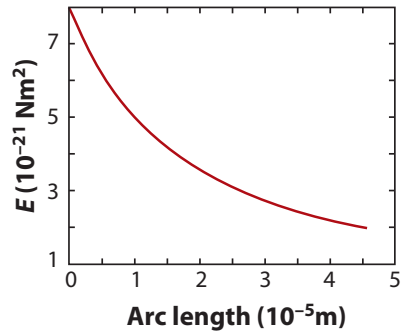

Figure 5

The moment balance of a planar swimming normozoospermic human cell moving with an approximately straight path in a highly viscous, non-Newtonian, $1 \%$ MC4000 (methylcellulose) solution, after migrating approximately $2 \mathrm{~cm}$ along a capillary tube at $37^{\circ} \mathrm{C}$. The focal plane is in the surface-accumulated layer and an equipotential of gravity, approximately $15 \mu \mathrm{m}$ from the inner surface of a 400- $\mu \mathrm{m}$-deep capillary tube. The medium has viscosity of $\mu=0.14 \mathrm{~Pa} \cdot \mathrm{s}$ and an elastic recoil time of $\lambda=6 \times 10^{-3} \mathrm{~s}$ on fitting rheological data to a Maxwell model, as presented by Smith et al. (2009c), where the experimental methods and kinematic data have previously been reported. (a) A time-lapse projection of the flagellar waveform onto the focal plane, with the sperm head trajectory noted in green. (b) A snapshot of the sperm, highlighting the digital capture of the (projected) flagellum. In general the two-dimensional projection exhibits slight length fluctuations, which do not exceed 10\% of the flagellum length (Smith et al. 2009c); the projected flagellum is truncated at $0.9 \mathrm{~L}$ to generate the images in panels $c-g$. The induced errors are discussed in the main text. (c) The curvature of this human sperm flagellum plotted as a function of time (horizontal axis) and arc length (vertical axis). An improved smoothing of the raw data entails that minor irregularities in the curvature plots of Smith et al. (2009c) are no longer present. $(d)$ The bending moment density due to hydrodynamic drag, $m_{b y d}$, calculated using viscoelastic resistive-force theory, via Equations 2 and 5 , with the vertical axis inherited from the plot shown in panel $c$ and analogous inheritance for the plots shown in panels $e$ and $g$. The bending stiffness of the sperm, $E(s)$, used to generate the plots shown in panels $e$ and $f$ is given in panel $b$ (see text for further details). (e) The bending moment due to the combination of active and passive internal sliding forces, by use of Equation 6. $(f)$ The bending moment density due to elastic bending stresses, by use of Equation 3. $(g)$ The differential shear, $\Delta(s)-\Delta(0)$, as defined in the caption of Figure 4, which is a measure of the extent of the amount of sliding undertaken by the flagellum. 
as hypothesized by Vernon \& Woolley (2004), Woolley et al. (2008), and Riedel-Kruse et al. (2007). Furthermore, the total sliding bending moment density is not uniformly dominated by hydrodynamic drag, even though the sperm is swimming within a medium over 100 times the viscosity of water; hence these observations indicate that the elastic resistance of the flagellum is highly significant.

One of the most interesting observations is that, although both the hydrodynamic and elastic bending moment densities have relatively complex profiles, the total sliding force bending moment density $m_{\text {slid }}(s, t)$ is approximately a collection of propagating traveling waves with relatively simple profiles and one characteristic frequency (Figures $5 \boldsymbol{d}-\boldsymbol{f}$ ). Furthermore, the differential shear, $\Delta(s)-\Delta(0)$ (see Figures $\mathbf{4}$ and $\mathbf{5 g}$ ), also has a simple traveling wave profile with the same characteristic frequency. An estimate of the maximal basal shearing, $\Delta(0)$, during motility with high curvature in the proximal flagellum is $170 \mathrm{~nm}$ for chinchilla sperm (Vernon \& Woolley 2004); these have a very compliant connecting piece and, in contrast to most mammals, exhibit sufficient basal sliding to be explicitly observed. This indicates that $\Delta(0)$ for the human sperm in Figure $\mathbf{5}$ is significantly smaller than $170 \mathrm{~nm}$; hence it is significantly smaller than the scale of $\Delta(s)-\Delta(0)$. Thus the differential shear is a good approximation for $\Delta(s)$ apart from very close to the sperm head. Hence, assuming that the passive sliding forces are monotonic functions of shearing with possibly a contribution from the derivatives of shear representing a viscous dissipative response, the passive sliding forces will also be waves inheriting the same propagation speed and characteristic frequency.

These plots therefore indicate that the characteristic distal meander in the flagellar form of the swimming human sperm in highly viscous media is generated by very simple patterns of dynein contraction along the flagellum, with a single characteristic frequency, rather than sophisticated mechanisms of dynein regulation. In addition, the fact that the peaks and troughs of total sliding force have approximately constant magnitude as the arc length is varied suggests a balance between changes in the passive response of the flagellum due to tapering of the outer dense fibers and fibrous sheath and the density of dynein contraction. Furthermore, the fact the elastic bending moment density in Figure $5 f$ only approximately shares the same traveling wave characteristics as the plot for curvature in Figure $5 \boldsymbol{c}$ suggests that the reduction in stiffness from the proximal to distal flagellum is dynamically important.

More generally, this simple example highlights the temporal and spatial resolution of microscopy, allowing the extraction of estimates for the densities of the hydrodynamic, elastic, and shear bending moments and forces. Such analyses may be useful in providing additional information to biologists researching a number of areas, for example, studies of the regulation of dynein in flagellar motility, which is characterized by numerous competing models. These include Brokaw's (1994, 1999, 2002) concept of curvature control, radial spoke regulation of dynein attachment as discussed by Lindemann \& Leisch (2010), dynein cross-bridge cycle regulation with active shear forces controlled by the sliding displacement via a self-organization model (Camalet \& Julicher 2000, Riedel-Kruse et al. 2007), and Lindemann's (1994a,b, 2004) geometric clutch control framework. Further examples where mechanical analyses at this resolution may be utilized include estimating the shearing forces within the flagellum induced by chemotactic or other stimulation and estimating the mechanical energy expenditure, particularly in the distal flagellum. The latter is of particular interest given the relative importance of ATP transport from mitochondria compared with local ATP generation in the distal flagellum, which is the subject of recent studies (for reviews and research, see Ford 2006, Takao \& Kamimura 2008, Storey 2008, Nascimento et al. 2008). 


\subsection{From In Vitro to In Vivo}

The long-term aim of relating theoretical and in vitro laboratory models to natural fertilization will require both new theory and physical, chemical, and molecular characterization of both the tract environment and sperm response. Examples include $(a)$ the rheology of cervical mucus and oviductal fluids, and how these vary during the menstrual cycle; (b) flagellar shear and bending mechanics; $(c)$ the release and diffusion of hormonal and other signals in the female reproductive tract; $(d)$ peristalsis and cilia-driven flow; (e) the factors and rates governing binding to and release from fallopian tube cells; $(f)$ the intracellular transduction of signals by sperm, including the processes of capacitation and chemotaxis; and finally $(g)$ sperm-zona penetration, for which enzyme lysis rates and diffusion coefficients are required. Hence there are many important avenues for future multidisciplinary research, with the eventual goal of modeling a virtual sperm capable of responding to the signals, insults, and microenvironments of an ambivalent female reproductive tract.

\section{SUMMARY AND FUTURE PERSPECTIVES}

Above we discuss the form and function of mammalian sperm, highlighting areas where mechanical analyses can enhance our understanding of these fascinating microswimmers, especially when utilizing the spatial and temporal resolution of digital microscopy. Despite extremely close biological parallels, the cutting edge of flagellar model construction has not reached the computational refinement achieved in a study of ciliated epithelium (Mitran 2007). This exemplary study proves the feasibility of discretely treating all the structural elements of the axoneme within a framework of nonlinear elasticity and yet couples this system to equations of rheological fluid dynamics, while performing large-scale simulations. On application to flagella, such approaches will allow the coupling of the exact physics of the fluid-flagellum interaction with classical research on the internal flagellar mechanics, including even more detailed structural biology as promised by atomic force microscopy (Ierardi et al. 2008). This integration across multiple scales will allow the investigation of current competing theories of beat pattern regulation as well as providing a means of investigating how molecular-level events dictate larger-scale flagellar behavior and sperm motility. While we have highlighted where mechanical studies can make an immediate impact in sperm motility, we close by postulating that longer-term research will be driven by such integrated, multiscale, sperm models combined with advances in three-dimensional, high-speed, live-cell imaging and intracellular signaling.

\section{SUMMARY POINTS}

1. Developments in the diagnosis and treatment of sperm submotility would have considerable impact on human fertility, animal breeding, and wildlife conservation.

2. The mammalian sperm has to navigate many different environments within the female reproductive tract with limited means of adaption.

3. Mechanics has an important role to play in understanding sperm motility in these diverse settings as sperm swimming ultimately emerges from the physical balance of hydrodynamic drag, the passive flagellar structural forces, and the sliding forces exerted by the internal molecular motors, the dyneins.

4. There is a hierarchy of tools, of increasing sophistication and accuracy but decreasing ease of implementation, from resistive-force theory, to slender-body theory, and direct numerical solutions to explore the hydrodynamics of sperm swimming. 
5. These tools often need to be coupled with models of the passive flagellum mechanical response and the internal molecular motors to explore the fundamental physics of sperm swimming, and further developing this field presents an important future challenge.

6. Considering such a modeling framework for sperm motility in the diverse regions of the female reproductive tract is underexplored and presents numerous applications and future investigations.

7. The combination of modeling and imaging also promises exciting developments, as illustrated by the extraction of estimates for the total internal sliding forces within the flagellum.

8. These studies promise advances in our understanding of the biophysics and mechanics associated with the response of a sperm's motility to its local environment. In turn, relating sperm swimming observed in the laboratory setting to motility behavior in vivo is an important but difficult challenge that would greatly improve fertility diagnostics and pharmacological screening for sperm (see the sidebar).

\section{DISCLOSURE STATEMENT}

The authors are not aware of any affiliations, memberships, funding, or financial holdings that might be perceived as affecting the objectivity of this review.

\section{ACKNOWLEDGMENTS}

EAG: This publication is based on work supported in part by Award KUK-C1-013-04, made by King Abdullah University of Science and Technology (KAUST). H.G. acknowledges the CAPES Foundation for sponsorship under contract grant BEX-4676/06-8. D.J.S. acknowledges the MRC for support under Special Training Fellowship G0600178; J.C.K.-B. and D.J.S. acknowledge support from Birmingham Science City, and previous support from the Wellcome Trust. The authors thank Dr. Nik Kapur of the University of Leeds for assistance with rheology and the Reproductive Biology and Genetics Group, University of Birmingham, for valuable comments.

\section{LITERATURE CITED}

Antman S. 2005. Non-Linear Problems of Elasticity. New York: Springer

Austin CR. 1995. Evolution of human gametes: spermatozoa. See Grudzinskas \& Yovich 1995, pp. 1-19

Baba SA, Mogami Y. 1985. An approach to digital image analysis of bending shapes of eukaryotic flagella and cilia. Cell Motil. Cytoskelet. 5(6):475-89

Baltz JM, Katz DF, Cone RA. 1988. Mechanics of sperm-egg interaction at the zona pellucida. Biophys. 7 . 54:643-54

Baltz JM, Williams PO, Cone RA. 1990. Dense fibers protect mammalian sperm against damage. Biol. Reprod. 43:485-91

Barratt CLR, Kay V, Oxenham SK. 2009. The human spermatozoon: a stripped down but refined machine. 7. Biol. $8: 63$

Barratt CLR, Kirkman-Brown J. 2006. Man-made versus female-made environment: Will the real capacitation please stand up? Hum. Reprod. Update 12:1-2

Bedford JM. 1998. Mammalian fertilization misread? Sperm penetration of the eutherian zona pellucida is unlikely to be a lytic event. Biol. Reprod. 59:1275-87 
Berke AP, Turner L, Berg HC, Lauga E. 2006. Hydrodynamic attraction of swimming microorganisms by surfaces. Phys. Rev. Lett. 101:038102

Blake JR. 1971. A note on the image system for a stokeslet in a no slip boundary. Proc. Camb. Philos. Soc. 70:303-10

Brennen C, Winet H. 1977. Fluid mechanics of propulsion by cilia and flagella. Annu. Rev. Fluid Mech. 9:339-98

Brokaw CJ. 1966. Effects of increased viscosity on the movements of some invertebrate spermatozoa. F. Exp. Biol. 45:113-39

Brokaw CJ. 1971. Bend propagation by a sliding filament model for flagella. 7. Exp. Biol. 55:289-304

Brokaw CJ. 1972. Computer simulation of flagellar movement I. Demonstration of stable bend propagation and bend initiation by the sliding filament model. Biophys. 7. 12:564-86

Brokaw CJ. 1975. Molecular mechanism for oscillation in flagella and muscle. Proc. Natl. Acad. Sci. USA 72:3102-6

Brokaw CJ. 1984. Automated methods for estimation of sperm flagellar bending parameters. Cell Motil. Cytoskelet. 4:417-30

Brokaw CJ. 1994. Control of flagellar bending: a new agenda based on dynein diversity. Cell Motil. Cytoskelet. 28:199-204

Brokaw CJ. 1999. Computer simulation of flagellar movement: VII. Conventional but functionally different cross-bridge models for inner and outer arm dyneins can explain the effects of outer arm removal. Cell Motil. Cytoskelet. 42:134-48

Brokaw CJ. 2002. Computer simulation of flagellar movement VIII: Coordination of dynein by local curvature control can generate helical bending waves. Cell Motil. Cytoskelet. 53:103-24

Brokaw CJ. 2003. Swimming with three-dimensional flagellar bending waves. Presented at Int. Symp. AquaBiomech., 2nd, Honolulu, HI

Brokaw CJ. 2008. Thinking about flagellar oscillation. Cell Motil. Cytoskelet. 66:425-36

Brokaw CJ. 2009. Simulation of cyclic dynein-driven sliding, splitting, and reassociation in an outer doublet pair. Biophys. 7. 97:2939-47

Burkman LJ. 1995. The motility of human spermatozoa before and after capacitation. See Grudzinskas \& Yovich 1995, pp. 122-39

Camalet S, Julicher F. 2000. Generic aspects of axonemal beating. New 7. Phys. 2:24

Childress S. 1981. Mechanics of Swimming and Flying. New York: Cambridge Univ. Press

Cortez R. 2001. The method of regularized stokeslets. SIAM J. Sci. Comput. 23:1204-25

Cortez R, Fauci L, Cowen N, Dillon R. 2004. Simulation of swimming organisms: coupling internal mechanics with external fluid dynamics. Comput. Sci. Eng. 6:38-45

Cosson J, Huitorel P, Gagnon C. 2003. How spermatozoa come to be confined to surfaces. Cell Motil. Cytoskelet. 54:56-63

Croxatto HB. 1995. Gamete transport. In Reproductive Endocrinology, Surgery and Technology, ed. J Adashi, Z Rosenwaks, pp. 395-98. New York: Raven

Croxatto HB. 2002. Physiology of gamete and embryo transport through the fallopian tube. Reprod. Biomed. Online 4:160-69

Cummings LM, Katz DF, Overstreet J, Hanson F. 1984. Sperm interactions in the penetration of cervical mucus. Proc. Am. Soc. Androl. 5:27

Cummins JM, Woodall PF. 1985. On mammalian sperm dimensions. 7. Reprod. Fertil. 75:153-75

Curry MR, Watson PF. 1995. Evolution of human gametes: spermatozoa. See Grudzinskas \& Yovich 1995, pp. $45-69$

Dam AHDM, Feenstra I, Westphal JR, Ramos L, van Golde RJT, Kremer JAM. 2007. Globozoospermia revisited. Hum. Reprod. Update 67:723-32

Dillon RH, Fauci LJ, Omoto C. 2003a. Mathematical modeling of axoneme mechanics and fluid dynamics in ciliary and sperm motility. Dyn. Contin. Discrete Impuls. Syst. A Math. Anal. 10:745-52

Dillon RH, Fauci LJ, Omoto C, Yang XZ. 2003b. Fluid dynamic models of flagellar and ciliary beating. Ann. N.Y. Acad. Sci. 1101:495-504

Dresdner RD, Katz DF. 1981. Relationships of mammalian sperm motility and morphology to hydrodynamic aspects of cell function. Biol. Reprod. 25:920-30
Illustrates Brokaw's modeling framework and how it provides insight into the mechanics of the inner and outer dynein arms. 
A seminal paper with the first extraction of the hydrodynamic forces on a sperm flagellum by combining modeling and imaging.

An elegant and informative theoretical and computational exploration of flagellum dynamics.
Drobnis EZ, Yudin AI, Cherr GN, Katz DF. 1988. Kinematics of hamster sperm during penetration of the cumulus cell matrix. Gamete Res. 21:367-83

Elfring GJ, Pak OS, Lauga E. 2010. Two-dimensional flagellar synchronization in viscoelastic fluids. F. Fluid Mech. 646:505-15

Fauci LJ, Dillon R. 2006. Biofluidmechanics of reproduction. Annu. Rev. Fluid Mech. 38:271-394

Fauci LJ, McDonald A. 1995. Sperm motility in the presence of boundaries. Bull. Math. Biol. 57:679-99

Fawcett DW. 1975. The mammalian sperm. Dev. Biol. 44:394-436

Fawcett DW. 1981. The Cell. Amsterdam: Elsevier Health Sci.

Florman HM, Jungnickel MK, Sutton KA. 2007. What can we learn about fertilization from cystic fibrosis? Proc. Natl. Acad. Sci. USA 104(27):11123-24

Friedrich BM, Riedel-Kruse IH, Howard J, Julicher F. 2010. High-precision tracking of sperm swimming fine structure provides strong test of resistive force theory. F. Exp. Biol. 213:1226-34

Ford WCL. 2006. Glycolysis and sperm motility: Does a spoonful of sugar help the flagellum go round? Hum. Reprod. Update 112:269-74

Fu HC, Wolgemuth CW, Powers TR. 2008. Beating patterns of filaments in viscoelastic fluids. Phys. Rev. E 78:041913

Fulford GR, Katz DF, Powell RL. 1999. Swimming of spermatozoa in a linear viscoelastic fluid. Biorbeology 35:295-309

Gadêlha H, Gaffney EA, Smith DJ, Kirkman-Brown JC. 2010. Migrating the model: Non-linear instabilities in flagellar dynamics may affect cell behaviour. F. R. Soc. Int. In press; doi: 10.1098/rsif.2010.0136

Gillies E, Cannon RM, Green RB, Pacey AA. 2009. Hydrodynamic propulsion of human sperm. F. Fluid Mech. 625:445-74

Gray J, Hancock GJ. 1955. The propulsion of sea urchin spermatozoa. F. Exp. Biol. 32:802-14

Green DPL. 1988. Sperm thrusts and the problem of penetration. Biol. Rev. 63:79-105

Grudzinskas JG, Yovich JL, eds. 1995. Gametes: The Spermatozoon. Cambridge, UK: Cambridge Univ. Press

Gueron S, Levit-Gurevich K, Liron N, Blum J. 1997. Cilia internal mechanism and metachronal coordination as the result of hydrodynamical coupling. Proc. Natl. Acad. Sci. USA 94:6001-6

Hancock GJ. 1953. The self-propulsion of microscopic organisms through liquids. Proc. R. Soc. Lond. A 217:96-121

Hilfinger A, Chattopadhyay AK, Julicher F. 2009. Nonlinear dynamics of cilia and flagella. Phys. Rev. E. 79:051918

Hines M, Blum JJ. 1978. Bend propagation in flagella I. Derivation of equations of motion and their simulation. Biophys. 7. 23:41-57

Hines M, Blum JJ. 1979. Bend propagation in flagella II. Incorporation of dynein cross-bridge kinetics into the equations of motion. Biophys. F. 25:421-42

Hines M, Blum JJ. 1983. Three-dimensional mechanics of eukaryotic flagella. Biophys. 7. 41:67-79

Hines M, Blum JJ. 1984. On the contribution of moment-bearing links to bending and twisting in a threedimensional sliding filament model. Biophys. 7. 46:559-65

Hines M, Blum JJ. 1985. On the contribution of dynein-like activity to twisting in a three-dimensional sliding filament model. Biophys. 7. 47:705-8

Hsu C, Dillon RH. 2009. A 3D motile rod-shaped monotrichous bacterial model. Bull. Math. Biol. 71:1228-63

Hunter RHF. 1995. Human sperm reservoirs and Fallopian tube function: a role for the intra-mural portion? Acta Obstet. Gynaecol. Scand. 74:677-81

Ierardi V, Niccolini A, Alderighi M, Gazzano A, Martelli F, Roberto S. 2008. AFM characterization of rabbit spermatozoa. Microsc. Res. Tech. 71:529-35

Immler S, Moore HDM, Breed WG, Birkhead TR. 2007. By hook or by crook? Morphometry, competition and cooperation in rodent sperm. PLoS One 2(1):e170

Johnson RE. 1980. An improved slender-body theory for Stokes flow. F. Fluid Mech. 99:411-31

Johnson RE, Brokaw CJ. 1979. Flagellar hydrodynamics: a comparison between resistive-force theory and slender-body theory. Biophys. F. 25:113-27

Julicher F, Prost J. 1997. Spontaneous oscillations of collective molecular motors. Phys. Rev. Lett. 78:4510-13

Katz DF, Drobnis EZ, Overstreet JW. 1989. Factors regulating mammalian sperm migration through the female reproductive tract and oocyte vestments. Gamete Res. 22:443-69 
Katz DF, Pedrotti L. 1977. Geotaxis by motile spermatozoa: hydrodynamic reorientation. F. Theor. Biol. 13:63-75

Katz DF, Phillips DM. 1986. The response of rhesus monkey sperm motility to cervical mucus and solid surfaces. Gamete Res. 13:231-39

Katz DF, Yanagimachi R. 1980. Movement characteristics of hamster spermatozoa within the oviduct. Biol. Reprod. 22:759-64

Kido A, Togashi K, Kataoka ML, Nakai A, Koyama T, Fujii S. 2008. Intrauterine devices and uterine peristalsis: evaluation with MRI. Magn. Reson. Imaging 26:54-58

Kim E, Yamashita M, Kimura M, Honda A, Kashiwabara S, Baba T. 2008. Sperm penetration through cumulus mass and zona pellucida. Int. F. Dev. Biol. 52:677-82

Kinukawa M, Ohmuro J, Baba S, Murashige S, Okuno M, et al. 2005. Analysis of flagellar bending in hamster spermatozoa: characterization of an effective stroke. Reproduction 72:1269-74

Kunz G, Beil D, Deininger H, Wildt L, Leyendecker G. 1996. The dynamics of rapid sperm transport through the female genital tract: evidence from vaginal sonography of uterine peristalsis and hysterosalpingoscintigraphy. Hum. Reprod. 11:627-32

Kunz G, Beil D, Huppert P, Leyendecker G. 2007. Oxytocin: a stimulator of directed sperm transport in humans. Reprod. Biomed. Online 14(1):32-39

Lai SK, Wang Y, Wirtz D, Hanes J. 2009. Micro- and macrorheology of mucus. Adv. Drug Deliv. Rev. 61:86-100

Lauga E, Powers TR. 2009. The hydrodynamics of swimming microorganisms. Rep. Prog. Phys. 72:096601

Leisch KA, Pelle DW, Dominic W, Lindemann CB. 2008. Insights into the mechanism of ADP action on flagellar motility derived from studies on bull sperm. Biophys. 7 . 95:472-82

Lighthill J. 1976. Flagellar hydrodynamics: JV Neumann lecture. SIAM Rev. 18:161-230

Lin Y, Mahan K, Lathrop WE, Myles DG, Primakoff P. 1994. A hyaluronidase activity of the sperm plasma membrane protein ph-20 enables sperm to penetrate the cumulus cell layer surrounding the egg. 7 . Cell Biol. 125:1157-63

Lindemann CB. 1994a. A geometric clutch hypothesis to explain oscillations of the axoneme of cilia and flagella. 7. Theor. Biol. 168:175-89

Lindemann CB. 1994b. A model of flagellar and ciliary functioning which uses the forces transverse to the axoneme as the regulator of dynein activation. Cell Motil. Cytoskelet. 29:141-54

Lindemann CB. 1996. Functional significance of the outer dense fibers of mammalian sperm examined by computer simulations with the geometric clutch model. Cell Motil. Cytoskelet. 34:258-70

Lindemann CB. 2002. Geometric clutch model version 3: the role of the inner and outer arm dyneins in the ciliary beat. Cell Motil. Cytoskelet. 52:242-54

Lindemann CB. 2004. Testing the geometric clutch hypothesis. Biol. Cell 96:681-90

Lindemann CB. 2009. Heart of the beat (the flagellar beat, that is). Biophys. 7. 97:2865-66

Lindemann CB, Leisch KA. 2010. Flagellar and ciliary beating: the proven and the possible. 7 . Cell Sci. 123:519-28

Lindemann CB, Mitchell DR. 2007. Evidence for axonemal distortion during the flagellar beat of chlamydomonas. Cell Motil. Cytoskelet. 64:580-89

Lindemann CB, Orlando A, Kanous KS. 1992. The flagellar beat of rat sperm is organized by the interaction of two functionally distinct populations of dynein bridges with a stable central axonemal partition. 7 . Cell Sci. 102:249-60

Liron N. 2001. The LGL (Lighthill-Gueron-Liron) theorem: historical perspective and critique. Math. Meth. Appl. Sci. 24:1533-40

Lopez-Garcia MDC, Monson RL, Haubert K, Wheeler MB, Beebe DJ. 2008. Sperm motion in a microfluidic fertilization device. Biomed. Microdevices 10:709-18

Machin KE. 1958. Wave propagation along flagella. F. Exp. Biol. 35:796-806

Mitchison TJ, Mitchison HM. 2010. How cilia beat. Nature 463:308-9

Mitran SM. 2007. Metachronal wave formation in a model of pulmonary cilia. Comput. Struct. 85:76374
An extensive review of the theory of cellular swimmers.

\section{Summarizes}

Lindemann's theory of the regulation of the flagellum beat.

A first study modeling flagellum elasticity, which indicated that force generation occurred within the flagellum and was confirmed later.

Focusing on cilia, an illustration of how one can model internal flagellum mechanics, coupled to viscoelastic media, in large-scale simulations. 
Murase M, Hines M, Blum JJ. 1989. Properties of an excitable dynein model for bend propagation in cilia and flagella. 7. Theor. Biol. 139:413-30

Nakanishi T, Isotani A, Yamaguchi R, Ikawa M, Baba T, et al. 2004. Selective passage through the uterotubal junction of sperm from a mixed population produced by chimeras of calmegin-knockout and wild-type male mice. Biol. Reprod. 71:959-65

Nascimento JM, Shi LZ, Tam J, Chandsawangbhuwana C, Durrant B, et al. 2008. Comparison of glycolysis and oxidative phosphorylation as energy sources for mammalian sperm motility, using the combination of fluorescence imaging, laser tweezers, and real-time automated tracking and trapping. 7 . Cell. Physiol. 217:745-51

Normand T, Lauga E. 2008. Flapping motion and force generation in a viscoelastic fluid. Phys. Rev. E 78:061907

Ohmuro J, Ishijima S. 2006. Hyperactivation is the mode conversion from constant-curvature beating to constant-frequency beating under a constant rate of microtubule sliding. Mol. Reprod. Dev. 73:1412-21

Okuno M, Asai DJ, Ogawa K, Brokaw CJ. 1981. Effects of antibodies against dynein and tubulin on the stiffness of flagellar axonemes. 7. Cell Biol. 91:689-94

Oldroyd JG. 1950. On the formulation of rheological equations of state. Proc. R. Soc. Lond. A 200:523-41

Olson GE, Linck RW. 1977. Observations of the structural components of flagellar axonemes and central pair microtubules from rat sperm. F. Ultrastruct. Res. 61:21-43

Olson SD, Suarez SS, Fauci LJ. 2010. A model of CatSper channel mediated calcium dynamics in mammalian spermatozoa. Bull. Math. Biol. In press; doi: 10.1007/s11538-010-9516-5

Pedley TJ, Kessler JO. 1992. Hydrodynamic phenomena in suspensions of swimming microorganisms. Annu. Rev. Fluid Mech. 24:313-58

Pelle DW, Brokaw CJ, Lesich KA, Lindemann CB. 2009. Mechanical properties of the passive sea urchin sperm flagellum. Cell Motil. Cytoskelet. 66:721-35

Powers TR. 2010. Dynamics of filaments and membranes in a viscous fluid. Rev. Mod. Phys. 82:1607-31

Pozrikidis C. 2002. A Practical Guide to Boundary Element Methods with the Software Library BEMLIB. Boca Raton, FL: CRC

Illustrates the combination of digital imaging and modeling to explore the mechanisms generating the flagellum beat.
Purcell EM. 1977. Life at low Reynolds number. Am. 7. Phys. 45:3-11

Riedel-Kruse IH, Hilfinger A, Howard J, Julicher F. 2007. How molecular motors shape the flagellar beat. HFSP F. 1:192-208

Rikmenspoel R. 1965. The tail movement of bull spermatozoa: observations and model calculations. Biophys. 7. $5: 365-92$

Rikmenspoel R. 1984. Movements and active moments of bull sperm flagella as a function of temperature and viscosity. F. Exp. Biol. 108:205-30

Rothschild L. 1963. Non-random distribution of bull spermatozoa in a drop of sperm suspension. Nature 198:1221-22

Sale WS, Satir P. 1977. Direction of active sliding of microtubules in tetrahymena cilia. Proc. Natl. Acad. Sci. USA 74:2045-49

Schmitz-Leisch KA, Lindemann CB. 2004. Direct measurement of the passive stiffness of rat sperm and implications to the mechanism of the calcium response. Cell Motil. Cytoskelet. 59:169-79

Seo D, Agca Y, Feng ZC, Critser JK. 2007. Development of sorting, aligning, and orienting motile sperm using microfluidic device operated by hydrostatic pressure. Microfluid. Nanofluid. 3:561-70

Shao B, Shi LZ, Nascimento JM, Botvinick EL, Ozkan M, et al. 2007. High-throughput sorting and analysis of human sperm with a ring-shaped laser trap. Microfluid. Nanofluid. 3:561-70

Shum H, Gaffney EA, Smith DJ. 2010. Modelling bacterial behaviour close to a no-slip plane boundary: the influence of bacterial geometry. Proc. R. Soc. Lond. A 466:1725-48

Sleigh MA. 1991. Hydrodynamic propulsion of human sperm. Protoplasma 164:45-53

Smith DJ, Blake JR. 2009. Surface accumulation of spermatozoa: a fluid dynamic phenomenon. Math. Sci. 34:74-87

Smith DJ, Gaffney EA, Blake JR. 2007. Discrete cilia modelling with singularity distributions: application to the embryonic node and the airway surface liquid. Bull. Math. Biol. 69:1477-510

Smith DJ, Gaffney EA, Blake JR. 2009a. Mathematical modelling of cilia-driven transport of biological fluids. Proc. R. Soc. Lond. A 465:2417-29 
Smith DJ, Gaffney EA, Blake JR, Kirkman-Brown JC. 2009b. Human sperm accumulation near surfaces: a simulation study. 7. Fluid Mech. 621:289-320

Smith DJ, Gaffney EA, Gadêlha H, Kapur N, Kirkman-Brown JC. 2009c. Bend propagation in the flagella of migrating human sperm, and its modulation by viscosity. Cell Motil. Cytoskelet. 66:220_ 36

Sobrero A, MacLeod J. 1962. The immediate postcoital test. Fertil. Steril. 13:184-89

Spagnolie SE, Lauga E. 2010. The optimal elastic flagellum. Phys. Fluids. 22:081902

Steptoe PC, Edwards RG. 1978. Birth after reimplantation of a human embryo. Lancet 38:366

Storey BT. 2008. Mammalian sperm metabolism: oxygen and sugar, friend and foe. Int. 7. Dev. Biol. 52:427-37

Suarez SS. 2002. Gamete transport. In Fertilization, ed. DM Hardy, pp. 3-28. New York: Academic

Suarez SS. 2008. Control of hyperactivation in sperm. Hum. Reprod. Update 14:647-57

Suarez SS. 2010. How do sperm get to the egg? Bioengineering expertise needed! Exp. Mech. In press; doi: $10.1007 /$ s11340-009-9312-z

Suarez SS, Dai X. 1992. Hyperactivation enhances mouse sperm capacity for penetrating viscoelastic media. Biol. Reprod. 46(4):686-91

Suarez SS, Pacey AA. 2006. Sperm transport in the female reproductive tract. Hum. Reprod. Update 12:23-37

Takao D, Kamimura S. 2008. FRAP analysis of molecular diffusion inside sea-urchin spermatozoa. F. Exp. Biol. 211:3594-600

Tam D. 2008. Motion at low Reynolds number. PhD thesis. Mass. Inst. Technol.

Taylor GI. 1951. Analysis of the swimming of microscopic organisms. Proc. R. Soc. Lond. A 209:447-61

Taylor GI. 1952. The action of waving cylindrical tails in propelling microscopic organisms. Proc. R. Soc. Lond. A 211:225-39

Taylor GI. 1960. G.I. Taylor's film clip illustrating the hydrodynamic reversibility of viscous flows. Available at http://www.physics.nyu.edu/pine/research/hydroreverse.html

Teran J, Fauci L, Shelley M. 2010. Viscoelastic fluid response can increase the speed and efficiency of a free swimmer. Phys. Rev. Lett. 104:038101

Turner RM. 2003. Tales from the tail: What do we really know about sperm motility? 7. Androl. 24(6):790-803

Vernon GG, Woolley DM. 2004. Basal sliding and the mechanics of oscillation in a mammalian sperm flagellum. Biophys. 7. 87:3934-44

Williams M, Hill CJ, Scudamore I, Dunphy B, Cooke ID, Barratt CLR. 1993. Sperm numbers and distribution within the human fallopian tube around ovulation. Hum. Reprod. 8:2019-26

Winet H, Bernstein GS, Head J. 1984. Observations on the response of human spermatozoa to gravity, boundaries and fluid shear. 7. Reprod. Fertil. 70:511-23

Wolf DP, Blasco L, Khan MA, Litt M. 1978. Human cervical mucus. IV. Viscoelasticity and sperm penetrability during ovulatory menstrual cycle. Fertil. Steril. 30(2):163-69

Woolley DM. 2003. Motility of spermatozoa at surfaces. Reproduction 126:259-70

Woolley DM. 2007. A novel motility pattern in quail spermatozoa with implications for the mechanism of flagellar beating. Biol. Cell 99:663-75

Woolley DM, Carter DA, Tilly GN. 2008. Compliance in the neck structures of the guinea pig spermatozoon, as indicated by rapid freezing and electron microscopy. 7. Anatomy 213:336-41

Woolley DM, Crockett RF, Groom WDI, Revell SG. 2009. A study of synchronisation between the flagella of bull spermatozoa, with related observations. F. Exp. Biol. 212:2215-23

Woolley DM, Vernon GG. 2001. A study of helical and planar waves on sea urchin sperm flagella, with a theory of how they are generated. F. Exp. Biol. 204:1333-45

Yanagimachi R. 1994. Mammalian fertilization. In The Physiology of Reproduction, ed. E Knobil, JD Neil, pp. 189-317. New York: Raven

Yaniv S, Jaffa A, Eytan O, Elad D. 2009. Simulation of embryo transport in a closed uterine cavity model. Eur. 7. Obstet. Gynecol. Reprod. Biol. 144:50-60

Yeung WSB, Ng VKH, Lay EYL, Ho PC. 1994. Human oviductal cells and their conditioned medium maintain the motility and hyperactivation of human spermatozoa in vitro. Hum. Reprod. 9:656-60

Youngren GK, Acrivos A. 1975. Stokes flow past a particle of arbitrary shape: numerical method of solution. 7. Fluid Mech. 69:377-403
Illustrates the resolution of sperm flagellum data capture, also demonstrating the importance of even small amounts of media viscoelasticity.

First computational simulation of finitelength, finiteamplitude swimming in a nonlinear viscoelastic fluid, showing how matching of beat frequency and relaxation time results in optimal progression. 
Yudin AI, Hanson FW, Katz DF. 1989. Human cervical mucus and its interaction with sperm: a fine-structural view. Biol. Reprod. 40:661-71

Yundt AP, Shack WJ, Lardner TJ. 1975. Applicability of hydrodynamic analyses of spermatozoan motion. 7. Exp. Biol. 62:27-41

\section{RELATED RESOURCES}

Blum JJ, Lubliner J. 1973. Biophysics of flagellar motility. Annu. Rev. Biophys. Bioeng. 2:181-219

Kolle S, Dubielzig S, Wehrend A, Konig P, Kummer W. 2009. Ciliary transport, gamete interaction, and effects of the early embryo on the oviduct: ex vivo analyses using a new digital videomicroscopic system in the cow. Biol. Reprod. 81:267-74 (The supplementary material provides additional illustrations of aspects of the sperm's journey in the cow's reproductive tract.)

Satir P, Christensen ST. 2007. Overview of structure and function of mammalian cilia. Annu. Rev. Physiol. 69:377-400

Webster DR, Weissburg MJ. 2009. The hydrodynamics of chemical cues among aquatic organisms. Annu. Rev. Fluid Mech. 41:73-90

Prof C.B. Lindemann's website: http://www2.oakland.edu/biology/lindemann/

Prof. C.J. Brokaw's website: http://www.cco.caltech.edu/ brokawc/ 


\section{舟}

Annual Review of Fluid Mechanics

Volume 43, 2011

Experimental Studies of Transition to Turbulence in a Pipe T. Mullin 1

Fish Swimming and Bird/Insect Flight

Theodore Yaotsu $W u$ .25

Wave Turbulence Alan C. Newell and Benno Rumpf

Transition and Stability of High-Speed Boundary Layers Alexander Fedorov .79

Fluctuations and Instability in Sedimentation Élisabeth Guazzelli and fohn Hinch

Shock-Bubble Interactions

Devesh Ranjan, Fason Oakley, and Riccardo Bonazza

Fluid-Structure Interaction in Internal Physiological Flows

Matthias Heil and Andrew L. Hazel

Numerical Methods for High-Speed Flows

Sergio Pirozzoli

Fluid Mechanics of Papermaking

Fredrik Lundell, L. Daniel Söderberg, and P. Henrik Alfredsson

Lagrangian Dynamics and Models of the Velocity Gradient Tensor in Turbulent Flows

Charles Meneveau

Actuators for Active Flow Control

Louis N. Cattafesta III and Mark Sheplak

Fluid Dynamics of Dissolved Polymer Molecules

in Confined Geometries

Michael D. Grabam

Discrete Conservation Properties of Unstructured Mesh Schemes

7. Blair Perot

Global Linear Instability

Vassilios Theofilis 
High-Reynolds Number Wall Turbulence

Alexander 7. Smits, Beverley 7. McKeon, and Ivan Marusic

Scale Interactions in Magnetohydrodynamic Turbulence

Pablo D. Mininni

Optical Particle Characterization in Flows

Cameron Tropea

Aerodynamic Aspects of Wind Energy Conversion

Jens Nørker Sørensen

Flapping and Bending Bodies Interacting with Fluid Flows

Michael 7. Shelley and fun Zhang

Pulse Wave Propagation in the Arterial Tree

Frans N. van de Vosse and Nikos Stergiopulos

Mammalian Sperm Motility: Observation and Theory

E.A. Gaffney, H. Gadêlha, D.7. Smith, F.R. Blake, and 7.C. Kirkman-Brown

Shear-Layer Instabilities: Particle Image Velocimetry Measurements and Implications for Acoustics

Scott C. Morris

Rip Currents

Robert A. Dalrymple, Famie H. MacMahan, Ad F.H.M. Reniers, and Varjola Nelko

Planetary Magnetic Fields and Fluid Dynamos

Chris A. Fones

Surfactant Effects on Bubble Motion and Bubbly Flows

Shu Takagi and Yoichiro Matsumoto

Collective Hydrodynamics of Swimming Microorganisms: Living Fluids

Donald L. Koch and Ganesh Subramanian

Aerobreakup of Newtonian and Viscoelastic Liquids

T.G. Theofanous

\section{Indexes}

Cumulative Index of Contributing Authors, Volumes 1-43

Cumulative Index of Chapter Titles, Volumes 1-43 699

\section{Errata}

An online log of corrections to Annual Review of Fluid Mechanics articles may be found at http://fluid.annualreviews.org/errata.shtml 